\title{
An Overview of the Geochemical Code MINTEQ: Applications to Performance Assessment for Low-Level Wastes
}
S. R. Peterson
B. E. Opitz
M. J. Graham
L. E. Eary

March 1987

Prepared for the U.S. Department of Energy under Contract DE-AC06-76RLO 1830

Pacific Northwest Laboratory Operated for the U.S. Department of Energy by Battelle Memorial Institute 


\title{
DISCLAIMER
}

This report was prepared as an account of work sponsored by an agency of the United States Government. Neither the United States Government nor any agency thereof, nor Battelle Memorial Institute, nor any of their employees, makes any warranty, expressed or implied, or assumes any legal liability or responsibility for the accuracy, completeness, or usefulness of any information, apparatus, product, or process disclosed, or represents that its use would not infringe privately owned rights. Reference herein to any specific commercial product, process, or service by trade name, trademark, manufacturer, or otherwise, does not necessarily constitute or imply its endorsement, recommendation, or favoring by the United States Government of any agency thereof, or Battelle Memorial Institute. The views and opinions of authors expressed herein do not necessarly state or reflect those of the United States Government or any agency thereof, or Battelle Memorial Institute.

\author{
PACIFIC NORTHWEST LABORATORY \\ operated by \\ BATTELI.E MEMORIAL INSTITUTE \\ for the \\ UNITED STATES DEPARTMENT OF ENERGY \\ under Contract DE-AC06-76RLO 1830
}

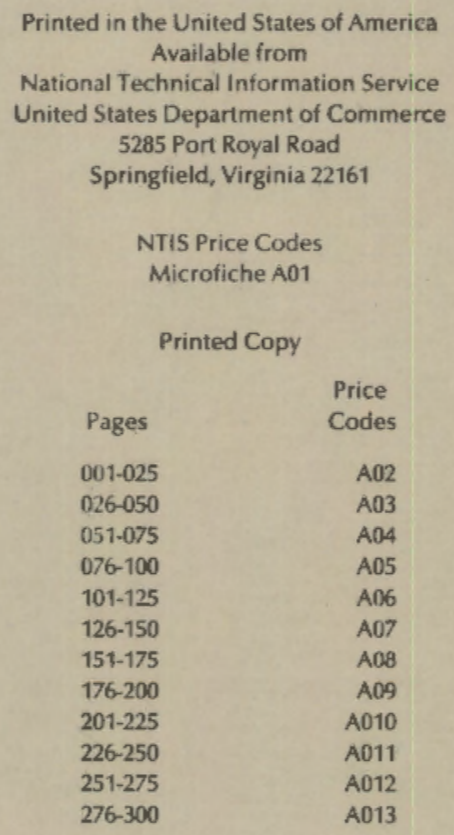


AN OVERVIEW OF THE GEOCHEMICAL CODE MINTEQ:

APPLICATIONS TO PERFORMANCE ASSESSMENT

FOR LOW-LEVEL WASTES

\author{
S. R. Peterson \\ B. E. Opitz \\ M. 3. Graham \\ L. E. Eary
}

March 1987

Prepared for

the U.S. Department of Energy

under Contract DE-ACO6-76RLO 1830

Pacific Northwest Laboratory

Richland, Washington 99352 


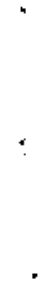

. 


\section{SUMMARY}

The MINTEQ geochemical computer code, developed at the Pacific Northwest Laboratory (PNL), integrates many of the capabilities of its two immediate predecessors, MINEQL and WATEQ3. The MINTEQ code will be used in the Special Waste Form Lysimeters-Arid program to perform the calculations necessary to simulate (model) the contact of low-level waste solutions with heterogeneous sediments or the interaction of ground water with solidified low-level wastes. The code can calculate ion speciation/solubility, adsorption, oxidation-reduction, gas phase equilibria, and precipitation/dissolution of solid phases.

Under the Special Waste Form Lysimeters-Arid program, the composition of effluents (leachates) from column and batch experiments, using laboratory-scale waste forms, will be used to develop a geochemical model of the interaction of ground water with commercial, solidified low-level wastes. The wastes being evaluated include power-reactor waste streams that have been solidified in cement, vinyl ester-styrene, and bitumen. The thermodynamic database for the code was upgraded preparatory to performing the geochemical modeling. Thermodynamic data for solid phases and aqueous species containing $\mathrm{Sb}, \mathrm{Ce}, \mathrm{Cs}$, or $\mathrm{Co}_{0}$ were added to the MINTEQ database. The need to add these data was identified from the characterization of the waste streams. The genchemical model developed from the laboratory data will then be applied to predict the release from a field-lysimeter facility that contains full-scale waste samples. The contaminant concentrations migrating from the waste forms predicted using MINTEQ will he compared to the long-term lysimeter data. This comparison will constitute a partial field validation of the geochemical model. 


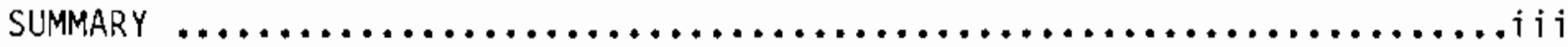

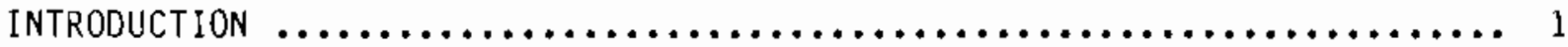

FEATURES OF MINTEQ CODE $\ldots \ldots \ldots \ldots \ldots \ldots \ldots \ldots \ldots \ldots \ldots \ldots \ldots \ldots \ldots \ldots$

SELECTION OF THE MINTEQ CODE $\ldots \ldots \ldots \ldots \ldots \ldots \ldots \ldots \ldots \ldots \ldots \ldots \ldots \ldots \ldots \ldots$

APPLICATIONS TO SPECIAL WASTE FORM LYSIMETERS -ARID PROGRAM .......... 19

SPECIATION AND SOLUBILITY........................... 19

MASS TRANSFER $\ldots \ldots \ldots \ldots \ldots \ldots \ldots \ldots \ldots \ldots \ldots \ldots \ldots \ldots \ldots \ldots \ldots \ldots \ldots \ldots \ldots$

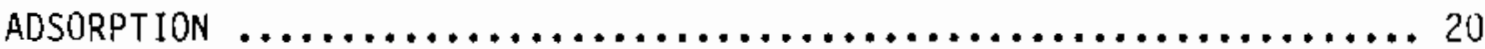

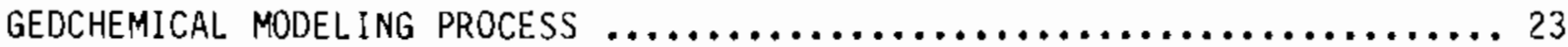

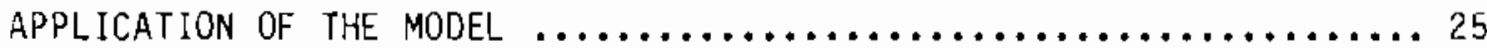

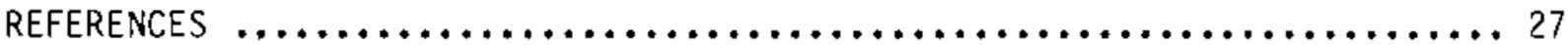

APPENDIX A - REACTIONS INVOLVING AQUEOUS SPECIES AND SOLID

PHASES OF ANTIMONY, CERIUM, CESIUM, AND COBALT ................. A.1 


\section{$\underline{\text { TABLES }}$}

1 Thermochemical Data for the Aqueous Species of Antimony .........7

2 Thermochemical Data for the Solid Phases of Antimony $\ldots . \ldots \ldots \ldots$.....

3 Thermochemical Data for the Aqueous Species of Cerium .......... 9

4 Thermochemical Data for the Solid Phases of Cerium ............. 10

5 Thermochemical Data for the Aqueous Species of Cesium .......... 11

6 Thermochemical Data for the Solid Phases of Cesium ............. 12

7 Thermochemical Data for the Aqueous Species of Cobalt .......... 14

8 Thermochemical Data for the Solid Phases of Cobalt ............ 15

9 Thermochemical Data for Auxiliary Species ................. 17

A.1 Reactions Involving Antimony Aqueous Species ....................

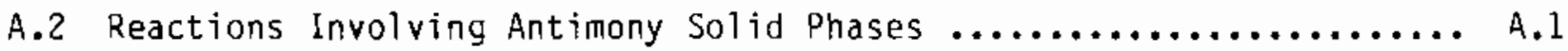

A.3 Reactions Involving Cerium Aqueous Species .......................

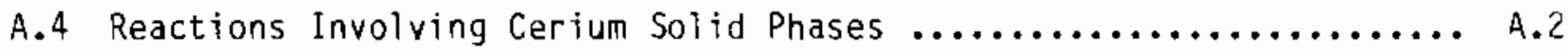

A.5 Reactions Involving Cesium Aqueous Species $\ldots \ldots \ldots \ldots \ldots \ldots \ldots \ldots \ldots$...

A.6 Reactions Involving Cesium Solid Phases ........................

A.7 Reactions Involving Cobalt Aqueous Species ................. A.4

A.8 Reactions Involving Cobalt Soljd Phases $\ldots \ldots \ldots \ldots \ldots \ldots \ldots \ldots \ldots$ 
INTRODUCT ION

The Special waste Form Lysimeters-Arid program was initiated in 1983 under the Low-Level Waste Management Program, for the long-term fieid evaluation of commercial, solidified, low-level wastes intended for shallow land burial (Graham and Walter 1984; Walter et a1. 1984). A similar task is being conducted at the Savannah River Laboratory for a humid climate. The primary objective of this program is to conduct waste-form leaching tests in a field facility to identify typical source terms generated by these wastes. The solidification agents being tested are cement, vinyl ester-styrene, and bitumen. The chemical reactions and physical processes that control the concentrations of radionuclides in the soil solution surrounding the waste in an arid climate are being identified.

In addition to the field monitoring, a series of laboratory testing and geochemical modeling exercises are under way. Standard leaching tests have been completed at Brookhaven National Laboratory. Column experiments are currently under way at Pacific Northwest Laboratory (PNL) to determine if the release of radionuclides from the waste is primarily controlled by diffusion through the waste form or by factors related to the adsorption of leachate onto the surrounding soil. The MINTEQ geochemical code will be used to develop an understanding of the contact of the low-level waste solutions with heterogeneous sediments and of the interaction of ground water with solidified, low-level wastes. MINTEQ will be used to model the waste stream, the waste form, and the column effluent from the field and laboratory experiments. Specifically, the goals of the laboratory and modeling work are to identify the mechanisms that control the release of radionuclides from the waste forms. Only reactions involving inorganic species will be considered in the modeling effort.

For the purposes of this report, a geochemical code is defined as the formulation in computer language of a mathematical model used to predict chemical behavior in geologic settings. One such code, MINTEQ, is being used on the Special waste Form Lysimeters-Arid program to develop an understanding of the chemical interactions that occur when ground water contacts commercial, lowlevel wastes and when leachates from these wastes contact the surrounding 
sediments in an arid environment. This report provides an overview of the MINTEQ geochemical code in the context of applications to performance assessments of shallow land burial of low-level wastes. Additionally, information on thermodynamic data for the solid and aqueous species of $\mathrm{Sb}, \mathrm{Ce}, \mathrm{Cs}$, and $\mathrm{Co}$ is given. The background of the Special Waste Form Lysimeters-Arid program ard the rationale for selecting MINTEQ are described, as is the geochemical modeling process, including examples of the application of MINTEQ to other problems. 
FEATURES OF THE MINTEQ CODE

The MINTEQ geochemical computer code was constructed by Felmy, Girvin and Jenne (1984) of PNL, who combined the best features of its two immediate predecessors, MINEQL (Westall et al. 1976) and WATEQ3 (Ba11 et al. 1981). MINTEQ can calculate ion speciation/solubility, adsorption, oxidation-reduction, gas phase equilibria, and precipitation/dissolution of solid phases. Moreover, MINTEQ can accept a finite inass for any solid considered for dissolution. Therefore, MINTEQ will continue to calculate the dissolution of the specified solid phase only until its initial mass is exhausted. MINTEQ is the on iy member of the REDEQL family of computer codes known to have this capability. [0ther members of this family of computer codes include REDEQL (Morel and Morgan 1972), REDEQL2 (MCDuff and More1 1973), GEOCHEM (Sposito and Mattigod 1980), and MINEQL (Westall et al. 1976).] This valuable feature enables MINTEQ to model flow-through systems. In such systems, the masses of solid phases that precipitate at earlier pore volumes can be dissolved at later pore volumes according to thermodynamic constraints imposed by the solution composition and solid phases present. It is imperative to have this feature incorporated into the computer code to perform the predictive geochemical modeing necessary to evaluate shallaw land burial sites. For a discussion of other geochemical computer codes, see Jenne (1981). 
. 
SELECTION OF THE MINTEQ CODE

Kincaid et al. (1984a, 1984b), after an exhaustive study of the existing geochemical computer codes, recommended MINTEQ and EQ3/EQ6 as the best codes available for modeling solute migration. From about 25 geochemical codes, they selected six for further evaluation on the basis of documented capabilities (Kincaid et al. 1984a). Five of these six codes were evaluated on their abilities to simulate solid-waste scenarios associated with deposits of fly ash, bottom ash, and flue gas desulfurization sludge (Kincaid et al. 1984b). Although these codes were not evaluated for low-level waste problems, the key aspects of the codes considered in the analysis are applicable to modeling lowlevel wastes. For the Special Haste Form Lysimeters-Arid program, the MINTEQ computer code was chosen over EQ3/EQ6 because it is more "user friendly" and has the ability to model adsorption reactions, something the current version of EQ3/EQ6 (Wolery 1984) cannot do. Adsorption reactions are important in many natural systems and in predicting the concentrations of contaminants in plumes migrating from shallow land burial sites.

Other important capabilities were considered in selecting the MINTEQ cOmputer code for potential application to evaluating the geochemistry of lowlevel waste in shallow land burial sites. For example, unlike certain codes [e.g., PHRFEQE (Parkhurst et al. 1980)], MINTEQ has the ability to automatically select and equilibrate a solution with the solids that are thermodynamically most stable in response to changes in solution composition. PHREEQE, in contrast, will not automatically consider all solids in the database that could enter into the equilibrium calculations. The MINTEQ computer program can also recompute the $\mathrm{pH}$ and pe (negative log of electron activity) as the solution composition changes in response to aqueous speciation and precipitation and dissolution of solid phases. The $\mathrm{pH}$ is computed from a modified form of the electroneutrality condition commonly termed the "proton condition" (Morel and Morgan 1972). The pe is calculated by initializing the mass totals for each oxidation state of an element and including a redox reaction between the components of each oxidation state. Alternatively, the pH and pe can also be fixed at the analytically measured values. Further details of the computational methods are provided by Felmy et al. (1984) and Westall et al. (1976). 
Finally, the completeness and credihility of the code's themodynamic database were important factors in the geochemical code selection. The thermodynamic database in MINTEQ was taken from the database of WATEQ3 and is well documented. The WATEQ3 database was developed and documented over a period of years by members of the U.S. Geological Survey (Truesdell and Jones 1974; Rall et al. 1980; Ball et a1. 1981). The MINTEQ database (Felmy et al. 1984) has recently been expanded using published critical reviews and studies of the aqueous species and solids of $\mathrm{Cr}$ (Schmidt 1984). In addition, preliminary compilations have been completed hy S. R. Peterson and others at PNL for the aqueous species and solids of $\mathrm{Mo}, \mathrm{Ra}, \mathrm{Se}$, Th, and $\mathrm{V}$, and for reduced sulfur species.

linder the Special Waste Form Lysimeters-Arid program, themodynamic data for the solid phases and aqueous species of $\mathrm{Sb}, \mathrm{Ce}, \mathrm{Cs}$, and $\mathrm{Co}$ were added to the MINTEO database. The need to add these data was identified from the characterization of the waste streams. Thermodynamic data for solid and aqueous species for $\mathrm{Sb}, \mathrm{Ce}, \mathrm{Cs}$, and $\mathrm{Co}$ are tabulated in Tables 1 to 8 . These tables list Gibb's free energies of formation $\left(\Delta G_{f}^{O}, 298\right)$, enthaipies of formation $\left(\Delta H_{f, 298}^{0}\right)$ and entropies $\left(S_{298}^{0}\right)$ for the solid phases and the aqueous species. Table 9 contains auxiliary thermodynamic data for the basis species used in formulating the chemical reactions. The references used are also listed in Tables 1 to 9. The reactions for aqueous and solid species for Sh, Ce, Cs, and Co, as fomilated for the MINTEQ datahase, are listed in Appendix A along with their associated values for $\log K_{298}$ and $\Delta H_{r, 298}^{0}$ values. Also inciuded in the Appendix Tables are the designated identification (ID) numbers for each of the aqueous species and solid phases. The ID numbers are seven-digit numbers. For aqueous complexes, the first three digits represent the ID number of the cationic component and the next three digits represent the anionic component. The last digit is an arbitrary designation. The first two digits of the In numbers for solids correspond to the mineral group and roughly follow the mineralogical classification in Dana's system (Dana and Ford 1957) and that described by Robie et al. (1978). The next three digits represent the leading cation in the chemical fommla and the last two digits are arbitrary designations. The data listed here have been calculated and formatted for the database of the geochemical computer code via the data processing code MCRT (Wolery 1984). 
TABLE 1. Thermochemical Data for the Aqueous Species of Antimony

\begin{tabular}{|c|c|c|c|c|}
\hline Formula & $\begin{array}{l}\Delta \mathrm{G}_{\mathrm{f}}^{0}, 298 \\
\mathrm{~kJ} / \mathrm{mol}\end{array}$ & $\begin{array}{l}\Delta \mathrm{H}_{\mathrm{f}, 298^{(\mathrm{a})}} \\
\mathrm{kJ} / \mathrm{mol} \\
\end{array}$ & $\begin{array}{l}S_{298}^{0}(\mathrm{a}) \\
\mathrm{J} / \mathrm{K} \cdot \mathrm{mol}\end{array}$ & References \\
\hline $\mathrm{Sb}_{2} \mathrm{~S}_{4}^{2-}$ & -99.500 & -219.200 & -52.300 & Wagman et a 1.1982 \\
\hline $\mathrm{SbO}^{+}$ & -177.110 & NA & NA & Wagman et a 1.1982 \\
\hline $\mathrm{SbO}_{2}^{-}$ & $-34 D .190$ & NA & NA & Wagman et al. 1982 \\
\hline $\mathrm{SbOF}^{\circ}$ & -487.400 & NA & NA & Wagman et al. 1982 \\
\hline $\mathrm{Sb}(\mathrm{OH})_{2} \mathrm{~F}^{\mathrm{O}}$ & -724.600 & NA & NA & Wagman et al. 1982 \\
\hline $\mathrm{Sh}(\mathrm{OH})_{3}^{\circ}$ & -644.700 & -773.600 & 116.300 & Wagman et al. 1982 \\
\hline $\mathrm{Sb}(\mathrm{OH})_{\overline{6}}^{-}$ & -1188.709 & $N A$ & NA & Baes and Mesmer 1976 \\
\hline
\end{tabular}
(a) Values unavailable for data listed as 'NA'. Species without available data for $\Delta G_{f}^{0} 298$ could not be added to the database because the log $K_{298}$ values could not be calculated.

The thermodynamic data selected in the compilations are primarily hased on accepted tabulations provided by such snurces as the U.S. National Bureau of Standards (Wagman et al. 1982), the committee on Data from Science and Technology of the International Council of Scientific Unions (CODATA Task Group 1978), and the U.S. Genlogical Survey (Robie et al. 1978). Some data, particularly for the cobalt sulfides, were taken from Vaughan and Craig (1978). Other sources of data include Cosovic et al. (1982) and Zhorov et al. (1976). Time constraints precluded a thorough review of the most recent journal literature on thermodynamic data for solids and aqueous species of Sb, Ce, C,s, and Co. In addition to the compilations, a limited number of journal articles were used to augment the thermodynamic data for these elements.

The data collected were not critically reviewed in the effort undertaken in the Special Waste Form Lysimeters-Arid program. Where values from sources differed, preference was given to data from compilations that had been critically reviewed and checked for internal consistency by the original authors. The following sources of data for the solid phases of Sb, Ce, Cs, and Co are listed in descending order of preference: 1) CODATA Task Group (1976, 1978), 2) Robie et al. (1978), 3) Wagman et al. (1982), and 4) Stull and Prophet (1971). Data from other sources were used only if data from these sources were 
TABLE 2. Thermochemical Data for the Solid Phases of Antimony

\begin{tabular}{|c|c|c|c|c|}
\hline Formula & $\begin{array}{l}\Delta \mathrm{G}_{\mathrm{f}, 298}^{0} \\
\mathrm{~kJ} / \mathrm{mol}\end{array}$ & $\begin{array}{c}\Delta \mathrm{H}_{\mathrm{f}}^{\mathrm{O}}, 298^{(\mathrm{a})} \\
\mathrm{kJ} / \mathrm{mol} \\
\end{array}$ & $\begin{array}{l}s_{298}(a) \\
J / x \cdot \text { mol }\end{array}$ & References \\
\hline $\mathrm{Sb}$ & 0 & 0 & 45.69 & Wagman et a1. 1982 \\
\hline $\mathrm{Sb}_{2} \mathrm{O}_{3}$ & -626.345 & -708.560 & 123.010 & Robie et al. 1973 \\
\hline $\mathrm{Sb}_{2} \mathrm{O}_{4}$ & -795.700 & $-907.50 n$ & 127.200 & Hagman et a1. 1982 \\
\hline $\mathrm{Sb}_{2} \mathrm{O}_{5}$ & -829.200 & -971.900 & 125.100 & Wagman et a l. 1982 \\
\hline $\mathrm{Sb}_{2} \mathrm{~S}_{3}$ & -173.600 & -174.900 & 182.000 & Robie et al. 1978 \\
\hline $\mathrm{Sb}_{4} \mathrm{O}_{6}$ Cubic & -1268.100 & -1440.600 & 220.900 & Hagman et al. 1982 \\
\hline $\mathrm{Sb}_{4} \mathrm{O}_{6}$ Orthorhombic & -1253.000 & -1417.100 & 246.1000 & Wagman et a1. $198 ?$ \\
\hline $\mathrm{SbCl}_{3}$ & -323.670 & -382.170 & 184.100 & Hagman et al. 1982 \\
\hline $\mathrm{SD}(\mathrm{OH})_{3}$ & -685.200 & NA & NA & Wagman et al. 1982 \\
\hline $\mathrm{SbBr}_{3}$ & -239.3 & -259.4 & 207.1 & Wagman et al. 1982 \\
\hline
\end{tabular}

(a) Values unavailable for data listed as 'NA'. Species without available data for $\Delta G_{f}^{0} 298$ could not be added to the database because the $\log K_{298}$ values could not be calculated.

not available. For aqueous data, the following primary sources are listed in descending order of preference: 1) CODATA Task Group (1976, 1978), 2) Wagman et a1. (1982), 3) Baes and Mesmer (1976), and 4) Smith and Martell (1975). Data were selected from other sources if information colld not be found for a particular species in any of these sources.

The above sources list data for standard state pressures of either 1 har or 1 atmosphere. Wagman et al. (1982) have pointed out that the effect of this small difference in standard state pressures on themochemical data for the condensed phases is negligibie, and hence the difference is ignored here.

In cases where sources, such as Smith and Martell (1976), give data as $\log K$ values for a specific reaction, $\Delta G_{f}^{0}, 298$ for a particular aqueous species or solid phase is calculated from

$$
\Delta G_{r, 298}^{O}=-[2.303 R(298)]\left(\log K_{298}\right)
$$

where $R=1.987 \times 10^{-3} \mathrm{kcal} / \mathrm{K} \cdot \mathrm{mol}$ 
TABLE 3. Thermochenical Data for the Aqueous Species of Cerium

\begin{tabular}{|c|c|c|c|c|}
\hline Fonmula & $\begin{array}{l}\Delta \mathrm{G}_{\mathrm{f}, 298}^{0} \\
\mathrm{~kJ} / \mathrm{mol}\end{array}$ & $\begin{array}{c}\Delta \mathrm{H}_{\mathrm{f}, 299^{(a)}}^{(\mathrm{a})} \\
\mathrm{kJ} / \mathrm{mol} \\
\end{array}$ & $\begin{array}{l}S_{298}^{0} \text { (a) } \\
\mathrm{J} / \mathrm{K} \cdot \mathrm{mo} ?\end{array}$ & References \\
\hline $\mathrm{CeBr} r^{2+}$ & -779.500 & NA & NA & Wagman et al. 1982 \\
\hline$C \mathrm{e}^{4+}$ & -503.800 & -537.200 & -301.000 & Wagman et a 1.1982 \\
\hline $\mathrm{CeC} 10_{4}^{2+}$ & -691.100 & -874.900 & -155.000 & Wagman et al. 1982 \\
\hline $\mathrm{CeF}^{2+}$ & -973.600 & NA & NA & Wagman et al. 1982 \\
\hline $\mathrm{CeNO}_{3}^{2+}$ & -789.500 & NA & NA & Wagman et al. 1982 \\
\hline $\mathrm{CeSO}_{4}^{+}$ & -1436.300 & -1590.800 & -71.000 & Wagman et al. 1982 \\
\hline $\mathrm{Ce}\left(\mathrm{SO}_{4}\right)_{2}$ & -2190.600 & -2493.200 & 8.000 & Wagman et al. 1982 \\
\hline $\operatorname{Cec} 1^{2+}$ & -798.7 & -840.6 & -88.3 & Wagman et al. 1982 \\
\hline$C e^{3+}$ & -672.0 & -696.2 & -205 & Wagman et al. 1982 \\
\hline
\end{tabular}

(a) Values unavailable for data listed as 'NA'.

The relationship

$$
\Delta G_{r, 298}^{0}=\Delta G_{f, 298}^{0}(\text { products })-\Delta G_{f, 298}^{0}(\text { reactants })
$$

was then used to calculate $\Delta G_{f, 298}^{0}$ for the unknown solid phase or aqueous species in the specific reactions. A consistent set of auxiliary data (Table 9) were used in such calculations. Gibh's free energies of formation taken from Naumov et al. (1974) were recalculated, when the $\log K_{298}$ for the associated reaction was given, by using the auxiliary data listed in Table 9.

Equilibrium constants are reported in compilations for solutions with particular ionic strengths. In the above calculations, where Gibb's free energies of formation for a particular solid phase or aqueous species were calculated from $\log K_{298}$, no attempt was made to correct the calculated values for ionic strength effects. Instead, ionic strengths are listed for the particular $\Delta G_{f, 298}^{0}$ values, and the data should be viewed with this fact in mind.

It should be noted that the themodynamic data assume that all reactions are written as formation reactions with either the solid or aqueous products on the right side. This convention is consistent with that used in MINTEQ. In 
TABLE 4. Thermochemical Data for the Solid Phases of Cerium

\begin{tabular}{|c|c|c|c|c|}
\hline Formula & $\begin{array}{l}\Delta G_{\mathrm{f}}^{0}, 298 \\
\mathrm{~kJ} / \mathrm{mol}\end{array}$ & $\begin{array}{c}\Delta H_{f, 298}^{0}{ }^{(a)} \\
\mathrm{kJ} / \mathrm{mol} \\
\end{array}$ & $\begin{array}{l}S_{2 g}^{0}(a) \\
\mathrm{J} / \mathrm{k} \cdot \mathrm{mol}\end{array}$ & References \\
\hline Ce & 0 & 0 & 72.0 & Wagman et al. 1982 \\
\hline $\mathrm{Ce}_{2} \mathrm{O}_{3}$ & -1706.2 & -1796.200 & 150.600 & Wagman et al. 1982 \\
\hline $\mathrm{Ce}_{2}\left(\mathrm{SO}_{4}\right)_{3} \cdot 8 \mathrm{H}_{2} \mathrm{O}$ & -5524.800 & NA & NA & Wagman et al. 1982 \\
\hline $\mathrm{CeCl}_{3}$ & -977.800 & -1053.500 & 151.000 & Wagman et a1. 1982 \\
\hline $\mathrm{Ce}\left(\mathrm{IO}_{3}\right)_{3} \cdot 2 \mathrm{H}_{2} \mathrm{O}$ & -1582.600 & NA & NA & Wagnan et al. 198? \\
\hline $\mathrm{CeO}_{2}$ & -1024.6 & -1088.7 & 62.300 & Wagman et a 1982 \\
\hline CeS & -451.500 & -459.400 & 78.200 & Wagman et a1. 1982 \\
\hline $\mathrm{CeCrO}_{3}$ & -1452.0 & -1540.0 & 105.0 & Wagman et al. 1982 \\
\hline
\end{tabular}

(a) Values thavailable for data listed as 'NA'. Species without available data for $\Delta \mathrm{G}_{\mathrm{f}}^{0} 298$ could not be added to the database because the $\log k_{298}$ values could not be calculated.

addition, reactions are written in terms of the basis species used in the MINTEQ code. For example, the formation of the aqueous complex $\mathrm{CoOH}^{+}$is written as

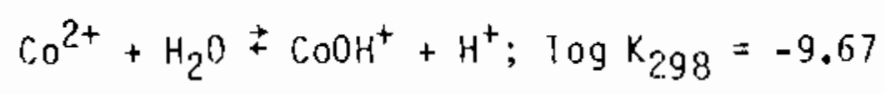

A version of the data processing code MCRT (bolery 1984) was used to check hand calculations of equilibrium constants for the formation reactions. MCRT calculates $\log K_{298}$ as $-\Delta G_{r, 298}^{0} /[2.303 R(298)]$.

MCRT provides a check of reaction stoichiometries, charge balance, and internal consistency of the thermochemical data. The internal consistency was checked where possible with the following expression:

$$
\Delta G_{f, 298}^{0}=\Delta H_{f, 298}^{0}-T \Delta 5: 298
$$

In a few cases, data were detemined to be inconsistent with respect to this equation. The authors of the compilations listing such data generally indicated that the free energy and enthalpy values were based on experimental 
TABLE 5. Themochenical Data for the Aqueous Species of Cesium

\begin{tabular}{|c|c|c|c|c|}
\hline Formula & $\begin{array}{r}\Delta G_{\mathrm{f}, 298}^{0} \\
\mathrm{~kJ} / \mathrm{mol} \\
\end{array}$ & $\begin{array}{c}\Delta H_{f}^{0}, 298^{(a)} \\
\mathrm{kJ} / \mathrm{mol} \\
\end{array}$ & $\begin{array}{l}S_{298}^{0}(a) \\
J / K \cdot m o l\end{array}$ & References \\
\hline $\mathrm{Cs}^{+}$ & -291.72 & -258.04 & 132.84 & COOATA Task Group 1978 \\
\hline $\mathrm{CsCl}^{\circ}$ & -421.08 & NA & NA & Smith and Martell 1976 \\
\hline
\end{tabular}

(a) Values unavailable for data listed as 'NA'.

measurements, whereas the entropies were estimated. In such cases, MCRT was used to calculate entropy values given the measured values for free energies and enthalpies of formation. It is these calculated values that are reported here for such cases.

MCRT also generates a set of correctly formatted files containing the assigned MINTEQ identification numbers and pertinent thermodynamic data for inclusion into the MINTEQ database.

The themodynamic data for the solids and aqueous species of Sb, Ce, Cs, and $C_{0}$ were added to the database hecause they are potentially important as migrating contaminants and, additionally, because it is important to have a thermodynamic database that is as complete as possihle. All components in the MINTEQ thermodynamic database are potentially important to low-level waste, even though a particular component (element or species) may not be a constituent of the initial waste. This is the case hecause the waste may react with surrounding sediments, indigenous migrating solutions, or solidification agents that do contain the specified component. This external component could then be important in the adsorption/ desorption and precipitation/dissolution reactions that affect the concentrations of components found in the un reacter low-level waste. 
TABLE 6. Themochemical Data for the Solid Phases of Cesium

\begin{tabular}{|c|c|c|c|c|}
\hline Formula & $\begin{array}{l}\Delta G_{\mathrm{f}, 298}^{0} \\
\mathrm{~kJ} / \mathrm{mol} \\
\end{array}$ & $\begin{array}{c}\Delta H_{\mathrm{f}, 298}^{0}{ }^{(\mathrm{a})} \\
\mathrm{kJ} / \mathrm{mol} \\
\end{array}$ & $\begin{array}{l}S_{298}^{0}(a) \\
\mathrm{J} / \mathrm{K} \cdot \mathrm{mol} \\
\end{array}$ & References \\
\hline $\mathrm{Cs}$ & 0 & 0 & 85.23 & Wagman et a 1.1982 \\
\hline $\mathrm{CsOH}$ & -370.702 & -415.722 & 98.742 & Robie et a1. 1978 \\
\hline $\mathrm{Cs}_{2} \mathrm{CO}_{3}$ & -1054.368 & -1139.722 & 204.472 & Wagman et a1. 1981 \\
\hline $\mathrm{Cs}_{2} \mathrm{Cr}_{2} \mathrm{O}_{7}$ & -1908.281 & -2088.820 & 330.076 & Wagman et a1. 1981 \\
\hline $\mathrm{Cs}_{2} \mathrm{O}$ & -308.361 & -346.812 & 146.858 & Robie et al. 1978 \\
\hline $\mathrm{Cs}_{2} \mathrm{SO}_{4}$ & -1323.818 & -1443.020 & 211.920 & Wagman et a 1.1981 \\
\hline $\mathrm{Cs}_{2} \mathrm{UO}_{4}$ & -1797.279 & -1919.996 & 219.660 & Robie et al. 1978 \\
\hline $\mathrm{CsAl}\left(\mathrm{SO}_{4}\right)_{2}\left(\mathrm{H}_{2} \mathrm{O}\right)_{12}$ & -5168.077 & -6094.833 & 686.092 & Wagman et al. 1981 \\
\hline $\operatorname{CsBr}$ & -391.413 & -405.806 & 113.052 & Wagman et al. 1981 \\
\hline $\mathrm{CsBr}_{3}$ & -405.011 & -432.626 & 221.752 & Wagman et a?. 1981 \\
\hline $\mathrm{CsBrO}_{3}$ & -284.972 & -375.807 & 163.594 & Wagman et al. 1981 \\
\hline $\mathrm{CsCl}$ & -414.551 & -443.044 & 101.169 & Wagman et al. 1981 \\
\hline $\mathrm{CsClO}_{3}$ & -307.942 & -411.706 & 156.063 & Wagman et al. 1981 \\
\hline $\mathrm{CsC} 10_{4}$ & -314.344 & -443.086 & 175.059 & Wagman et al. 1981 \\
\hline $\mathrm{CsCuCl}_{3}$ & -627.182 & -698.728 & 213.384 & Wagman et al. 1981 \\
\hline CsF & -525.510 & -553.543 & 92.801 & Wagman et al. 1981 \\
\hline $\mathrm{CsHF}_{2}$ & -858.975 & -923.827 & 135.185 & Wagman et a1. 1981 \\
\hline $\operatorname{CsI}$ & -340.578 & -346.603 & 123.051 & Wagman et a1. 1981 \\
\hline $\mathrm{CsI}_{2} \mathrm{Br}$ & -385.346 & -397.062 & 238.488 & Wagman et al. 1981 \\
\hline $\mathrm{CsI}_{3}$ & -354.803 & -361.916 & 235.141 & Wagman et a1. 1981 \\
\hline $\mathrm{CsI}_{4}$ & -357.732 & -365.682 & 290.788 & Wagman et al. 1981 \\
\hline $\mathrm{CsIBr}_{2}$ & -422.584 & NA & NA & Wagman et a1. 1981 \\
\hline CsIBrCl & -443.504 & NA & NA & Wagman et al. 1981 \\
\hline CstBrF & -546.012 & NA & NA & Wagman et a1. 1981 \\
\hline $\mathrm{CsIO}_{3}$ & -433.881 & NA & NA & Wagman et al. 1981 \\
\hline
\end{tabular}

(a) Values unavailable for data listed as 'NA'. Species without available data for $\Delta G_{f}^{0} 298$ could not be added to the database because the $\log K_{298}$ vatues could not be calculated. 
TABLE 6. (contd)

\begin{tabular}{|c|c|c|c|c|}
\hline Formula & $\begin{array}{r}\Delta \mathrm{G}_{\mathrm{f}}^{0}, 298 \\
\mathrm{~kJ} / \mathrm{mol} \\
\end{array}$ & $\begin{array}{c}\Delta H_{f}^{0}, 298^{(a)} \\
\mathrm{kJ} / \text { mol } \\
\end{array}$ & $\begin{array}{l}S_{298}^{0}(a) \\
\mathrm{J} / \mathrm{K} \cdot \mathrm{mol}\end{array}$ & References \\
\hline $\mathrm{CsIO}_{4}$ & -380.744 & NA & NA & Wagman et a 1. 1981 \\
\hline $\mathrm{CsNO}_{3}$ & -406.601 & -505.971 & 155.226 & Wagman et. a?. 1981 \\
\hline $\mathrm{CsBO}_{2}$ & -915.1 & -972.4 & 104.35 & Wagman et al. 1982 \\
\hline $\mathrm{CsBCl} 4$ & -826.7 & -941.8 & 151.0 & Wagman et al. 1982 \\
\hline
\end{tabular}

(a) Values unavailable for data listed as 'NA'. Species without available tata for $\Delta G_{f}^{0}, 298$ could not be added to the database because the $\log _{298}$ values could'not be calculated. 
TARI.E. 7. Thermochemical Data for the Aqueous Species of Cobalt

\begin{tabular}{|c|c|c|c|c|}
\hline Formula & $\begin{array}{l}\Delta G_{f}^{0}, 298 \\
\mathrm{~kJ} / \mathrm{mol}\end{array}$ & $\begin{array}{c}\Delta H_{f}^{0}, 298^{(a)} \\
\mathrm{kJj} / \mathrm{mol}^{\circ} \\
\end{array}$ & $\begin{array}{l}s_{298}^{0}(a) \\
\mathrm{J} / \mathrm{K} \cdot \mathrm{mol}\end{array}$ & References \\
\hline $\mathrm{Co}^{2+}$ & -54.392 & -58.199 & -112.968 & Robie et al. 1978 \\
\hline $\mathrm{CO}_{2} \mathrm{OH}^{3+}$ & -281.583 & NA & NA & Sinith and Martel 1976 \\
\hline $\mathrm{CO}^{\overline{3}+}$ & 134.014 & 92.006 & $-305.892^{(b)}$ & Robie et ai. 1978 \\
\hline $\mathrm{Co}_{4}(\mathrm{OH})_{4}^{4+}$ & -993.282 & NA & NA & Smith and Martell 1976 \\
\hline $\mathrm{CoCO}_{3}^{\circ}$ & -600.404 & NA & NA & Cosovic et al. 1982, $I=0.56$ \\
\hline $\mathrm{CoF}^{+}$ & -338.486 & NA & NA & Smith and Martell 1976 \\
\hline $\mathrm{CoHCO}_{3}^{+}$ & -649.189 & NA & NA & Zhorov et al. $1976, I=0.7$ \\
\hline $\mathrm{COHPO}$ & -1156.123 & NA & NA & Smith and Martels 1976, I $=0.5$ \\
\hline $\mathrm{CoHS}^{+}$ & -79.496 & NA & NA & Smith and Martell 1976 \\
\hline $\mathrm{Co}(\mathrm{HS})_{2}^{0}$ & -80.333 & NA & NA & Naumov et al. 1974 \\
\hline $\mathrm{Co}\left(\mathrm{NH}_{3}\right)^{2+}$ & -92.466 & -145.185 & 12.552 & Wagman et al. 1982 \\
\hline $\mathrm{Co}\left(\mathrm{NH}_{3}\right)_{2}^{2+}$ & -127.612 & NA & NA & Wagman et al. 1982 \\
\hline $\mathrm{Co}\left(\mathrm{NH}_{3}\right) \frac{2+}{3}+$ & -158.992 & NA & NA & Wagman et al. 1982 \\
\hline $\mathrm{Co}\left(\mathrm{NH}_{3}\right)_{4}^{2+}$ & -189.117 & NA & NA & Wagman et al. 1982 \\
\hline $\mathrm{Co}\left(\mathrm{NH}_{3}\right)_{5}^{2+}$ & -216.313 & NA & NA & Smith and Martell 1976, $T=293 \mathrm{~K}$ \\
\hline$\left[\mathrm{Co}\left(\mathrm{NH}_{3}\right)_{5}\right] \mathrm{Cl}^{2+}$ & -291.625 & -628.018 & 341.414 & Wagman et al. 1982 \\
\hline$\left[\mathrm{CO}\left(\mathrm{NH}_{3}\right)_{5}\left(\mathrm{NO}_{2}\right)\right]^{2+}$ & -167.360 & -613.374 & 163.176 & Wagman et al. 1982 \\
\hline $\mathrm{Co}\left(\mathrm{NH}_{3}\right)_{6}^{3+}$ & -156.900 & -584.923 & 146.022 & Wagman et al. 1982 \\
\hline$\left[\mathrm{Co}\left(\mathrm{NH}_{3}\right)_{6}\right] \mathrm{Br}^{2+}$ & -252.714 & -718.393 & 163.176 & wagman et al. 1982 \\
\hline$\left[\mathrm{Co}\left(\mathrm{NH}_{3}\right)_{6}\right] \mathrm{Cl}^{2+}$ & -279.910 & -770.274 & 117.152 & Wagman et al. 1982 \\
\hline$\left[\mathrm{Co}\left(\mathrm{NH}_{3}\right)_{6}\right] \mathrm{I}^{2+}$ & -202.924 & -648.938 & 213.384 & 'Nagman et al. 1982 \\
\hline$\left[\mathrm{Co}\left(\mathrm{NH}_{3}\right)_{6}\right] \mathrm{OH}^{2+}$ & -330.536 & NA & NA & ragman et al. 1982 \\
\hline$\left[\mathrm{Co}\left(\mathrm{NH}_{3}\right)_{6}\right] \mathrm{SO}_{4}^{+}$ & -920.898 & -1492.433 & 238.070 & Wagman et al. 1982 \\
\hline $\mathrm{COOH}^{+}$ & -236.396 & NA & NA & Smith and Martell 1976 \\
\hline $\mathrm{CO}(\mathrm{OH})_{2}^{\mathrm{O}}$ & -421.705 & NA & NA & Wagman et al. 1982 \\
\hline $\mathrm{CO}(\mathrm{OH}) \frac{2}{3}$ & -581.994 & NA & NA & Smith and Martell 1976 \\
\hline $\mathrm{CO}(\mathrm{OH})_{4}^{2-}$ & -741.823 & NA & NA & Smith and Martell 1976 \\
\hline $\mathrm{CoP}_{2} \mathrm{O}_{7}^{2-}$ & -2008.320 & NA & NA & smith and Martell 1976 \\
\hline $\mathrm{CoS}_{2} \mathrm{O}_{3}^{\circ}$ & -471.955 & NA & NA & Strith and Martell 1976, I = 0.1 \\
\hline $\mathrm{HCoO}_{2}^{-}$ & -407.522 & NA & NA & Wagman et a1. 1982 \\
\hline
\end{tabular}

(a) Values unavajlable for data listed as 'NA'.

(b) Calculated by MCRT. 
TABLE 8. Thermochemical Data for the Solid Phases of Cobalt

\begin{tabular}{|c|c|c|c|c|}
\hline Formula & $\begin{array}{l}\Delta G_{f}^{0}, 298 \\
\mathrm{~kJ} / \mathrm{mol}\end{array}$ & $\begin{array}{c}\Delta H_{f}^{0}, 298^{(a)} \\
\mathrm{kJ} / \mathrm{mol}\end{array}$ & $\begin{array}{c}S_{298}^{0}{ }^{(a)} \\
\mathrm{J} / \mathrm{K} \cdot \mathrm{mol}\end{array}$ & References \\
\hline Co, $\alpha$ hexagonal & 0.000 & 0.000 & 30.041 & Robie et al. 1978 \\
\hline $\mathrm{CoSO}_{4} \cdot 7 \mathrm{H}_{2} \mathrm{O}$ & -2473.42 & -2979.93 & 406.06 & Wagman et a . 1982 \\
\hline $\mathrm{CO}_{3}\left(\mathrm{AsO}_{4}\right)_{2}$ & -1620.8 & NA & NA & Wagman et al. 1982 \\
\hline $\mathrm{CO}_{3}\left(\mathrm{PO}_{4}\right)_{2}$ & -2398.6 & NA & NA & Wagman et al. 1982 \\
\hline $\mathrm{Co}_{3} \mathrm{O}_{4}$ & -772.868 & -891.192 & 102.508 & Robie et a1. 1978 \\
\hline $\mathrm{CoC}_{2}$ & -269.8 & -312.5 & 109.16 & Wagman et al. 1982 \\
\hline $\mathrm{CoCl}_{2} \cdot 2 \mathrm{H}_{2} \mathrm{O}$ & -764.835 & -922.990 & 188.280 & Wagman et al. 1982 \\
\hline $\mathrm{CoCl}_{2} \cdot 6 \mathrm{H}_{2} \mathrm{O}$ & -1725.482 & -2115.430 & 343.088 & Wagman et al. 1982 \\
\hline $\mathrm{COCl}_{2} \cdot \mathrm{H}_{2} \mathrm{O}$ & -527.184 & -615.048 & 191.209 & Wagman et al. 1982 \\
\hline $\mathrm{CoF}_{2}$ & -647.265 & -692.034 & 81.965 & Wagman et al. 1982 \\
\hline $\mathrm{CoHPO}_{4}$ & -1181.9 & NA & NA & Wagman et al. 1982 \\
\hline$\left[\mathrm{Co}\left(\mathrm{NH}_{3}\right)_{5} \mathrm{Cl}\right] \mathrm{Cl} l_{2}$ & -582.5 & -1017.1 & 366.100 & Wagman et al. 1982 \\
\hline$\left[\mathrm{Co}\left(\mathrm{NH}_{3}\right)_{5} \mathrm{H}_{2} \mathrm{O}\right] \mathrm{Cl}_{3}$ & -777.8 & -1288.0 & 346.0 & Wagman et al. 1982 \\
\hline $\mathrm{Co}\left(\mathrm{NH}_{3}\right)_{5} \mathrm{NO}_{2}\left(\mathrm{NO}_{3}\right)_{2}$ & -412.9 & -1088.7 & 331.0 & Wagman et a1. 1982 \\
\hline $\mathrm{Co}\left(\mathrm{NH}_{3}\right)_{6} \mathrm{Br}_{3}$ & -500.9 & -1002.90 & 325.1 & Wagman et al. 1982 \\
\hline $\mathrm{Co}\left(\mathrm{NH}_{3}\right)_{6}\left(\mathrm{ClO}_{4}\right)_{3}$ & -221.1 & -1034.7 & 615.0 & Wagman et al. 1982 \\
\hline $\mathrm{Co}\left(\mathrm{NH}_{3}\right)_{6}\left(\mathrm{NO}_{3}\right)_{3}$ & -524.5 & -1282.0 & 448.0 & Wagman et al. 1982 \\
\hline $\mathrm{CoO}$ & -214.179 & -237.944 & 52.969 & Robie et al. 1978 \\
\hline $\mathrm{CO}(\mathrm{OH})_{2}{ }^{(b)}$ & -450.198 & NA & NA & Wagman et al. $198 ?$ \\
\hline $\mathrm{CO}(\mathrm{OH})_{2}(\mathrm{C})$ & -454.382 & -539.736 & 79.496 & Wagman et al. 1982 \\
\hline $\mathrm{CO}(\mathrm{OH})_{2}^{(\mathrm{d})}$ & -458.148 & NA & NA & Wagman et a1. 1982 \\
\hline $\mathrm{Co}(\mathrm{OH})_{2}(\mathrm{e})$ & -459.82 & NA & NA & Naumov et al. 1974 \\
\hline $\mathrm{CO}(\mathrm{OH})_{3}$ & -596.638 & -730.526 & 83.680 & Latimer 1952 \\
\hline $\mathrm{CoSO}_{4}$ & -782.3 & -888.3 & 118.0 & Wagman et a1. 1982 \\
\hline $\mathrm{CoSO}_{4} \cdot 6 \mathrm{H}_{2} \mathrm{O}$ & -2235.36 & -2683.6 & 367.61 & Wagman et a1. 1982 \\
\hline
\end{tabular}

\footnotetext{
(a) Values unavailable for data listed as 'NA'.

(b) Blue precipitate.

(c) Pink precipitate.

(d) Pink precipitate, aged.

(e) Transvaalite.
} 


\section{TABLE 8. (contd)}

\begin{tabular}{|c|c|c|c|c|}
\hline Formula & $\begin{array}{l}\Delta G_{f}^{0}, 298 \\
\mathrm{~kJ} / \mathrm{mol}^{\prime}\end{array}$ & $\begin{array}{c}\Delta H_{f}^{0}, 298 \\
\quad \mathrm{KJ} / \mathrm{mol} \\
\end{array}$ & $\begin{array}{l}S_{298}(\mathrm{a}) \\
\mathrm{J} / \mathrm{K} \cdot \mathrm{mol}\end{array}$ & References \\
\hline $\mathrm{CoSO}_{4} \cdot \mathrm{H}_{2} \mathrm{O}$ & -1042.946 & -1200.724 & 175.728 & Naumov et al. 1974 \\
\hline $\mathrm{CoS}_{2}$ & -145.603 & -153.134 & 69.036 & Vaughan and Craig 1978 \\
\hline $\mathrm{Cog}_{9} \mathrm{~S}_{8}$ & -834.708 & -851.026 & $471.955^{(b)}$ & Vaughan and Craig 1978 \\
\hline $\mathrm{CO}_{3}\left(\mathrm{AsO}_{4}\right)_{2} \cdot 8 \mathrm{H}_{2} \mathrm{O}$ & -3518.744 & NA & NA & Naumov et al. 1974 \\
\hline $\mathrm{COOOH}$ & -398.735 & NA & NA & Sillen and Martell 1964 \\
\hline $\cos$ & -95.395 & $-96.23 ?$ & 58.576 & Vaughan and Craig 1973 \\
\hline $\mathrm{Co}_{3} \mathrm{~S}_{4}$ & -348.946 & -358.987 & 184.514 & Vaughan and Craig 1978 \\
\hline CoAs & -49.371 & -51.045 & 59.413 & Naumov et al. 1974 \\
\hline $\mathrm{COAs}_{2}$ & -96.650 & -83.262 & 146.440 & Naumov et al. 1974 \\
\hline $\mathrm{CoCO}_{3}$ & -640.152 & -716.092 & 38.617 & Naumov et aT. 1974 \\
\hline
\end{tabular}

(a) Values unavailable for data listed as 'NA'.

(b) Calculated by MCRT. 
TABLE 9. Thermochemical Data for Auxiliary Species

\begin{tabular}{|c|c|c|c|c|}
\hline Formula & $\begin{array}{l}\Delta G_{\mathrm{f}, 298}^{0} \\
\mathrm{kCal} / \mathrm{mol}\end{array}$ & $\begin{array}{l}\Delta H_{f}^{0}, 298 \\
\mathrm{kcal} / \text { mol }\end{array}$ & $\begin{array}{c}\mathrm{S}_{298}^{0} \\
\mathrm{cal} / \mathrm{K} \cdot \mathrm{mol} \\
\end{array}$ & References \\
\hline $\mathrm{H}_{2} \mathrm{O}$ & -56.69 & -68.32 & 16.72 & Robie et al. 1978 \\
\hline $\mathrm{F}^{-}$ & -67.34 & -80.15 & -3.15 & Robie et al. 1978 \\
\hline $\mathrm{CI}^{-}$ & -31.38 & -39.93 & 13.56 & Robie et al. 1978 \\
\hline $\mathrm{Br}^{-}$ & -24.87 & -29.04 & 19.80 & Robie et a1. 1978 \\
\hline $\mathrm{CrO}_{4}^{-}$ & -2.06 & -30.91 & 43.50 & Wagman et a 1.1982 \\
\hline $\mathrm{HS}^{-}$ & 2.88 & 4.20 & 15.00 & Robie et a1. 1978 \\
\hline $\mathrm{S}_{2} \mathrm{O}_{3}^{2-}$ & -122.80 & -155.90 & 8.90 & Naumov et a 1974 \\
\hline $\mathrm{SO}_{4}^{2-}$ & -177.97 & -217.32 & 4.80 & Robie et a .1978 \\
\hline $\mathrm{CO}_{3}^{2-}$ & -126.17 & -161.84 & -13.60 & Robie et al. 1978 \\
\hline $\mathrm{P}_{4}^{3-}$ & -243.50 & -305.30 & -53.00 & Robie et a1. 1978 \\
\hline $\mathrm{H}_{3} \mathrm{AsO}_{4}^{\circ}$ & -183.10 & -215.70 & 44.00 & Wagman et al. 1982 \\
\hline $\mathrm{NO}_{2}^{-}$ & -8.9 & -25.0 & 33.5 & Robie et al. 1978 \\
\hline $\mathrm{NO}_{3}^{-}$ & -26.61 & -49.57 & 35.00 & Robie et a 1.1978 \\
\hline $\mathrm{NH}_{4}^{+}$ & -18.99 & -31.85 & 26.57 & Wagman et al. 1982 \\
\hline $\mathrm{H}^{+}$ & 0.000 & 0.000 & 0.000 & By Definition \\
\hline $\mathrm{I}^{-}$ & -12.412 & -13.599 & 25.500 & CODATA 1976 \\
\hline $\mathrm{A} 1^{3+}$ & -116.900 & -126.910 & -73.610 & Robie et al. 1978 \\
\hline $\mathrm{Cu}^{2+}$ & 15.720 & 15.700 & -23.200 & Cowan 1986 \\
\hline$v 0_{2}^{2+}$ & -227.700 & -243.500 & -23.200 & Langmuir 1978 \\
\hline $\mathrm{CrO}_{4}^{2-}$ & -174.810 & -210.930 & 13.780 & Schmidt 1984 \\
\hline $\mathrm{H}_{3} \mathrm{BO}_{3}^{\circ}$ & -231.560 & -256.290 & 38.800 & Wagman et a?. 1968 \\
\hline $\mathrm{Mn}^{2+}$ & -19.600 & -27.000 & -63.980 & Plismmer et al. 1976 \\
\hline $\mathrm{MoO}_{4}^{2-}$ & -199.900 & -238.500 & 5.500 & Wagman et al. 1969 \\
\hline$v 0_{2}^{+}$ & -140.300 & -155.300 & -10.100 & Wagman et al. 1971 \\
\hline$v 0^{2+}$ & -106.700 & -116.300 & -32.00 & Wagman et al. 1968 \\
\hline $\mathrm{Mg}^{2+}$ & -108.700 & -111.580 & -33.000 & Parker et al. 1971 \\
\hline $\mathrm{Cr}(\mathrm{OH})_{2}^{+}$ & -153.350 & -177.290 & -10.030 & Schmidt 1984 \\
\hline$e^{-}$ & 0.000 & 0.000 & 15.604 & CODATA 1976 \\
\hline
\end{tabular}




\section{APPLICATIONS TO SPECIAL WASTE FORM LYSIMETERS-ARID PROGRAM}

For the modeling associated with the Special Waste Form Lysimeters-Arid program, the MINTEQ computer code can be considered to have four parts: 1) a speciation submodel, 2) a solubility submodel, 3) a mass transfer submodel, and 4) an adsorption submodel. The calculations completed by each submodel are dependent on the thermodynamic data stored in the MINTEQ database. Based on a need identified from thorough characterization of the wastes being evaluated in the program, thermodynamic data for $\mathrm{Sb}, \mathrm{Ce}, \mathrm{Cs}$, and $\mathrm{Co}$ were added to the database. Because of their interrelationship, the ion speciation and solubility submodels will be discussed together.

SPECIATION AND SOLUBILITY

In the speciation submodel, MINTEQ computes the activities of complexed and uncomplexed cationic and anionic species, neutral ion pairs, and the activities of cationic and anionic redox species. These activities are then used in the solubility submodel, which performs solubility calculations in which ion activity products for solids and minerals are calculated. The calculated activity products (AP) are compared to the solubility products (K) of minerals and solids stored in the thermodynamic database of MINTEQ to develop a saturation index $[\log (\mathrm{AP} / \mathrm{K})]$. This saturation index indicates the degree of undersaturation or oversaturation of a solution relative to solids and minerals of interest. If a solution is oversaturated with respect to a particular solid phase, the value of the saturation index $[\log (A P / K)]$ is greater than 0 . Oversaturation conditions are usualiy explained by precipitation kinetics and/or mineralogical factors that prevent the solid from precipitating at a rate sufficient to control the concentrations of its dissolved components. If the saturation index reflects undersaturation $[\log (\mathrm{AP} / \mathrm{K})$ is less than 0$]$, it is concluded that either I) a less soluble (thermodynamically more stable) solid is controlling the dissolved constituents' concentrations, 2) another mechanism, such as adsorption, is controliing the concentrations of the component species below their solubility products, or 3) the constituents' concentrations in the source are low. A consideration of the saturation indices of the soiutions resulting from the interactions of low-level waste with aqueous solutions 
will provide useful information as to solids, minerals, and mechanisms that control or affect the concentrations of specified constituents of the system. It will then be possible, using this information, to make predictions about future environmental concentrations of the constituents.

\section{MASS TRANSFER}

The results from the solubility submodel of MINTEQ are used by the mass transfer submodel to calculate the mass of solid that precipitates or dissolves. MINTEQ keeps track of the mass of solid that precipitates from solution or dissolves from the sediment. The solids that represent the solution are those considered likely to precipitate from solution because of changes that occur in specific solution parameters (e.g., JH and pe) when the solution comes into contact with the assemblage of solid phases (chemical model) that represent the waste forms. If the solubility submodel indicates that a given solution is oversaturated with respect to a solid phase considered in the chemical model of the system, the mass transfer submodel will precipitate that solid until the solution is at equilibrium $[i . e ., \log (\log A P / K)=0]$ with the solid. If the solubility submodel indicates that a solution is undersaturated with regard to a particular solid in the chemical model of the system, the MINTEQ mass transfer submodel will dissolve that solid until solution equilibrium is achieved with respect to the solid, or until the finite mass of the solid is completely dissolved.

\section{ADSORPTION}

Adsorption of a solute molecule on the surface of a solid can involve removing the solute molecule from the solution, removing solvent from the solid surface, and attaching the solute to the surface of the solid (Stum and Morgan 1981). The MINTEQ computer code can model adsorption onto solid surfaces via several mechanisms: 1) a distribution coefficient, Kd, 2) a Langmuir isotherm, 3) a Freundlich isotherm, 4) an ion exchange model, 5) a constant-capacitance surface complexation model, and 6) a triple-layer surface complexation model. 
The $k_{d}$ is defined as the ratio of the mass of adsorbed species to the concentration of the species remaining in solution. Adsorption modeis conveniently describe the adsorption of solutes by solids at constant temperature (thus the label "isotherm") in quantitative terms (Bohn et al. 1979). The Langmuir isotherm has the advantage over the $K_{d}$ (linear isotherm) in considering a mass balance on surface adsorption sites (Felmy et al. 1984). The Freundich isotherm was derived empirically from adsorption data for dilute solutions. The Freundich isotherm implies that the energy of adsorption decreases logarithmically as the fraction of surface covered increases (Bohn et a1. 1979). In the MINTEQ code, the activities of the aqueous species that dominate the adsorption reaction with the surface sites are used instead of the total concentrations of the adsorbate. The use of the activities instead of concentrations allows consideration of the "effective" concentration of each adsorbate. Consequently, the $K_{d}$ 's and Langmuir and Freundlich isotherms are referred to as "activity" $K_{d}$ 's and "activity" Langmuir and Freundlich isotherms. Ion exchange reactions involve the exchange of ions with like charge on the solid surface. The two surface complexation models include treatment of electrostatic effects due to surface charge and the effect of soiution chemistry on the solid. A general description of each algorithm is given by Felmy et al. (1984). Additional details on the mathematical formulation and nuances of the aigorithms can be obtained from references cited by felmy et al. (1984). 


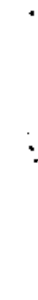


GEOCHEMICAL MODELING PROCESS

A chemical model of the interaction of low-level wastes with sediments and solutions indigenous to the shallow land burial site can be developed through many different methods and thought processes. Plurmer (1984) has conveniently classified the modeling approach into "inverse" and "forward" methods. The inverse method, as described by Plummer, is similar to the inverse problem in hydrology (Freeze and Cherry 1979). The goal of both the inverse and forward methods is to identify the dominant mechanisms that account for concentration distributions of aqueous and solid phases along a flow path. These mechanisms involve such things as precipitation/dissolution of solids, adsorption/desorption, ion exchange, and advection-dispersion. Once the dominant mechanisms are identified and incorporated into a chemical model of the system, the chemical model can be used in the forward mode to predict aqueous concentrations and mass transfers in as yet unstudied systems. In this report, the term "inverse method" is used narrowly to mean a method that considers only mass transfers between two points such that the analytical data at the final point are replicated exactiy. Any other method is considered to be a forward method.

The inverse method of modeling is more quantitative than the forward method. However, it can be employed only when appropriate hydrogeochemical data along the evolution-path (i.e., the flow path along which mass transfer mechanisms operate) are available. With the inverse method, one works from observed data at two points separated in space along a single flow path. The objective is to find a reaction model that produces mass transfers along the flow path that are consistent with the data at the two observed points. The inverse method is not constrained by thermodynamics; however, the results should be checked by the forward method using thermodynamic constraints on the plausible phases seiected for the conceptual chemical model of the system. Minimally, ion speciation and solubility calculations should be perforined on the initial and final points in the system to check thermodynamic feasibility. In other words, the inverse method predicts the mass transfers that are consistent with the geochemistry of the initial and final observation points. These mass transfers are unconstrained by thermodynamic considerations. 
A simple mass balance computer code, such as BALANCE (Parkhurst et al. 1982) is sufficient to develop a model using the inverse method. The column experiments conducted at PNL will provide the data necessary to perform the inverse modeling. The two points to be modeled are the chemistry of the batch leachate entering the soil column and the chemistry of the leachate after it passes through the column.

The forward method uses a developed conceptual chemical model of the system to predict the aqueous compositions and mass transfers resulting from the sediment/solution interaction. This is the only practical approach in the absence of analytical data sufficient for inverse nodeling. The conceptual chemical model of the system may or may not be constrained thermodynamically. Even if it is not constrained thermodynamically, the forward method differs from the inverse method hy being used in a predictive mode. One may develop a chemical model using the inverse method and subsequentiy use the chemical model in a forward mode to predict water quality data and mass transfers in the future or at another site. To use thermodynamic constraints in making predictions regarding a site, the forward method is implied. This use of thermodynamic constraints is both an advantage and liability of the forward method. We gain a better thermodynamic understanding of the system, but it is difficult to consider the rate-dependent or kinetic effects. The inverse method leads to field-derived kinetic data. The kinetic data are field derived in the sense that any reactions that might occur are inerged in the postulated mass transfer reactions that produce the measured aqueous phase compositions at the final point in the evolution-path.

Using either the forward or the inverse method, one tries to develop a conceptual chemical model of the system, hereafter referred to as a chemical model. Depending on the method selected, the chemical model is used to replicate exactly or predict as closely as possible the observed or future analytical data at a point. Using the forward method, one could develop a generic chemical model of the interactions of low-level wastes with geologic sediments that could be used in a predictive mode at other sites; this would preclude the necessity of developing an individual chemical model for each site. The forward method is the only alternative at sites where sufficient and appropriate 
data are unavailable. Use of the forward method would enable a regulatory agency to evaluate a potential site for suitability as a low-level waste burial site. The inverse method can only be used at existing sites to develop a reaction model to account for what has been observed at the site. However, this reaction model could then be used in a forward mode to predict future conditions at the site.

For the Special Waste Form Lysimeters-Arid program, the geochemical model will be developed using the laboratory data and employing both the inverse and forward methods. Once developed, the model will be used in the forward mode to predict the release from the field-lysimeter facility containing full-scale waste samples. The contaminant concentrations migrating from the wastes will be predicted and compared to the long-term lysimeter data. This will constitute a partial field validation of the geochemical model.

APPLICATION OF THE MODEL

Peterson et al. (1983) were among the first to use a hypothesized solidphase assemblage to predict the aqueous compositions resulting from a solution contacting heterogeneous sediments in a flow-through system. The solids and minerals in this solid-phase assemblage were allowed to precipitate and dissolve as the solution composition changed. The solid-phase assemblage, in conjunction with the MINTEQ geocherical computer code, was used to simulate the contact of acid uranium mill tailings solutions from wyoming with indigenous geologic sediments containing calcium carbonate. The compositions of the aqueous phase and the change in mass of each solid phase in the assemblage were calculated as successive pore volumes of solution interacted with the solidphase assemblage. This generic approach has been successful in predicting contaminant concentrations in plumes migrating from uranium mill tailings waste impoundments, and it is currently being used to evaluate migration from coal combustion by-products. The MINTEO computer code will be a useful tool for better understanding the interaction of low-level wastes with the surrounding environments, and in developing a chemical model of these interactions. This 
chemical model, partially validated under the Special Waste Form LysimetersArid program, will be valuable in helping evaluate the migration of radionuclides from solidified low-level waste at shallow land burial sites. 
REFERENCE.S

Baes, C.F., and R. F. Mesmer. 1976. The Hydrolysis of Cations. Wiley, New York.

Ba11, J. W., D. K. Nordstron, and E. A. Jenne. 1980. Additional and Revised The mochemical Data and Computer Code for WATEQ2-A Computerized Chernical Model for Trace and Major Element Speciation and Mineral Equilibria of Natural Waters. Water Resources Investigations WRI-78-116, U.S. Geological Survey, Washington, D.C.

Ba11, J. W., E. A. Jenne, and M. W. Cantrell. 1981. HATEQ3: A Geochemical Model with Uranium Added. Open-File Report 81-1183, U.S. Geological Survey, Washington, D.C.

Bohn, H. L., B. L. MeNeal, and G. A. O'Connor. 1979. Soil Chemistry. Wiley, New York.

CODATA Task Group. 1976. "Recommended Key Values for Themodynamics, 1975." J. Chem. The rmodynamics 8:603-605.

CODATA Task Group. 1978. "CODATA Recommended Key Values for Themodynamics, 1977. Report of the CODATA Task Group on Key Values for The modynamics, 1977." J. Chem. The modynamics 10:903-906.

Cosovic B., D. Degobbis, H. Bilinski, and M. Branica. 1982. "Inorganic Cohalt. Species in Seawater." Geoch. Cosmo. Acta 46:151-158.

Cowan, C. F. 1986. "A Methodology for Determining Dose-Response Toxicity Models Incorporating the Effects of Chemical Speciation." Dissertation, University of Washington, Seattle, Washingtnn.

Dana, E. S., and W. E. Ford. 1957. A Textbook of Mineralogy. Wiley, New York.

Felmy, A. R., D. C. Girvin, and E. A. Jenne. 1984. MINTEQ: A Computer Program for Calculating Aqueous Geochemical Equilibria. (NTIS PB84-157148) EPA-600/3-84-032, National Technical Information Service, Springfield, Virginia.

Freeze, R. A., and J. A. Cherry. 1979. Groundwater. Prentice-Hall, Englewood Cliffs, New Jersey.

Graham, M. J., and M. B. Walter. 1984. "Evaluation of the Performance of Solidified Commercial Low-Level Wastes in an Arid Climate." In Proceedings of the Sixth Annual DOE Low-Level Waste Management Program Participant's Information Meeting, CONF-8409115, National Technical Information Service, Springfield, Virginia. 
Jenne, E. A. 1981. Waste/Rock Interactions Technology Program Geochemical Modeling: A Review. PNL-3574, Pacific Northwest Lahoratory, Richland, Washington.

Kincaid, C. T., J. R. Morrey, and J. E. Rogers, 1984a. Geohydrochemical Models of Solute Migration, Volume 1. EA-3417, Electric Power Research Institute, Palo Alto, California.

Kincaid, C. T., J. R. Morrey, S. B. Yabusaki, A. R. Felmy, and J. E. Rogers. 1984b. Geohydrochemical Models for Solute Migration, Volume 2: Preliminary Evaluation of Selected Computer Codes. EA-3417, Electric Power Research Institute, Palo Alto, California.

Langmir, D. 1978. "Uranium Solution-Mineral Equilibria at Low Temperatures with Applications to Sedimentary Ore Deposits." Geoch. Cosmo. Acta 42:547569.

Latimer, W. M. 1952. The Oxidation States of the Elements and Their Potentials in Agueous Solutions. 2nd ed. Prentice-Hall, Englewood Cliffs, New Jersey.

McDuff, R. E., and F. M. M. Morel. 1973. Description and Use of the Chemical Equilibrium Program REDEQL2. EQ-73-02, Califormia Institute of Technology, Pasadena, California.

Morel, F., and J. Morgan. 1972. "A Numerical Method for Computing Fquilibria in Aqueous Systems." Environ. Sci. Technol. 6:56-67.

Naumov, F. B., B. N. Rhyzhenko, and I. L. Khodakovsky. 1974. Handhook of Thermodynamic Data. Trans1. G. J. Soleimani, eds. I. Barnes and U. Spaltz. Report No. ISSGS-WPD-74-001, National Technical Information Service, Springfield, Virginia.

Parker, V. B., D. D. Wagman, and W. H. Evans. 1971. Selected Values of Chemical Thermodynamic Properties. National Bureau of Standards Technical Note 270-6, IJ.S. Govt. ?rinting Office, Washington, D.C.

Parkhurst, D. L., D. C. Thorstenson, and L. N. Plumer. 1980. PHREEQE - A Computer Program for Geochernical Calculations. Water Resources Investigations 80-96, II.S. Geologica! Survey, Washington, D.C.

Parkhurst, D. L., L. N. Plummer, and D. C. Thorstenson, 1982, BALANCE- A Computer Program for Calculating llass Transfer for Geochemical Reactions in Groundwater. Water Resources Investigations 32-14, U.S. Geological Survey, Wastington, D.C.

Peterson, S. R., A. R. Felmy, R. J. Serne, and G. W. Gee. 1983. Predictive Genchemical Modeling of Interactions Between Uranium Mill Tailings Solutions and Sediments in a Flow-Through System: Model Formulations and Preliminary Results. NUREG/CR-478? (PNL 4732), II.S. Nuclear ReguTatory Commission, Washingt on, D.C. 
Plummer, L. N. 1984. "Principles of Geochemical Modelling." Paper presented at the Canadian-American Conference on the Practical Applications of Groundwater Geochemistry, June 22-26, 1984, Banff, Alberta, Canada.

Plummer, L. N., B. F. Jones, and A. H. Truesde11. 1976. WATEQF - A Fortran IV Version of WATEQ, A Computer Program for Calculating Chemical Equilibrium of Natural Waters. Water Resources Investigations WRI-76-13, IJ.S. Geological Survey, Washington, O.C.

Robie, R. A., B. S. Hemingway, and F. R. Fisher. 1978. Thermodynamic Properties of Minerals and Related Substances at $298.15 \bar{K}$ and 1 Bar $(105$ Pascals) Pressure and at Higher Temperatures, U.S. Geological Survey Bulletin 1452, reprinted with corrections 1979 , 13.S. Government Printing office, Washington, D.C.

Schmidt, R. L. 1984. Themodynamic Properties and Environmental Chemistry of Chromium. PNL-4881, Pacific Northwest Laboratory, Richland, Washington.

Sillen, L. G., and A. E. Martel1. 1964. Stability Constants of Metal-Ion Complexes. Spec. Puh. 17, Chemical Society, London.

Smith, R. M., and A. E. Martel1. 1976. Critical Stability Constants, Vol. 4: Inorganic Complexes. Plenur Press, New York.

Sposito, G., and S. V. Mattigod. 1980. GEOCHEM: A Comuter Program for the Calculation of Chemical Equilibria in Soil Solutions and Other Natural ivater Systems. University of California, Riverside, California.

Stul1, D. R., and H. Prophet. 1971. JANAF Thermochemical Tables: National Reference Data Series. V. 37. U.S. Bureau of Standards, U. S. Government Printing of fice, Washington, D.C.

Stumm, W., and J. J. Morgan. 1981. Aquat ic Chemistry, An Introduction Emphasizing Chemical Equilibria in Natural Waters. 2nd ed. Wiley, New York.

Truesdell, A. H., and B. F. Jones, 1974. "WATEQ, A Computer Prograin for CalCulating Chemical Equilibria of Natural Naters". U.S. Geol. Survey J. Res. $2: 233-274$.

Vaughan, D. J., and J. R. Craig. 1973. Mineral Chemistry of Metal Sulfides. Cambridge University Press, New York.

Wagman, ก. D., W. H. Evans, V. B. Parker, I. Halow, S. M. Bailey, and R. H. Schumm. 1968. Selected Values of Chemical Thermodynamic Properties. National Bureau of Standards Technical Note 270-3, 11. S. Govt. Printing office, Washington, n.r.

Wagman, D. D., W. H. Evans, V. B. Parker, I. Halow, S. M. Bailey, and R. H. Schumm. 1969. Selected Values of Chemical Thermodynamic Properties. National Rureau of Standards Technical Note 270-4, 11.S. Govt. Printing office, 'dashington, D.C. 
Wagman, D. D., W. H. Evans, V. B. Parker, I. Halow, S. M. Bailey, R. H. Schumm, and K. L. Churney. 1971. Selected Values of Chemical The rmodynarnic Properties. National Bureau of Standards Technical Note 270-5, J.S. Govt. Printing Office, Washington, D.C.

Wagman, D. D., W. H. Evans, V. R. Parker, R. H. Schumrn, and R. L. Nuttall. 1981. Selected Values of Chemical Thermodynamic Properties: Compounds of Uranium, Protactinium, Thorium, Actinium, and the Alkali Metals. National Bureau of Standards Technical Note 270-8, 11.S. Govt. ?rinting Office, Washington, D.C.

Wagman, D. D., W. H. Evans, V. B. Parker, R. H. Schumm, I. Halow, S. M. Bailey, K. L. Churney, and R. L. Nuttal1. 1982. "The NBS Tables of Chemical Thermodynamic Properties. Selected Values for Inorganic and $\mathrm{Cl}$ and $\mathrm{C} 2$ Organic Substances in SI Units." J. Phys. Chem. Ref. Data 11 (Supplement No. 2):1-39?.

Walter, M. B., M. J. Graham, and G. W. Gee. 1984. A Field Lysimeter Facility for Evaluating the Performance of Commercial Solidified Low-level Waste. PNL-5253, Pacific Northwest Laboratory, Richland, Washington.

Westall, J.C., J. L. Zachary, and F. M. More?. 1976. "MINEQL: A Computer Program for the Calculation of Chemical Equilibrium Composition of Aqueous Systems." Tech. Note 18, Dept, of Civil Engineering, Massachusetts Institute of Technology, Cambridge, Massachusetts.

Wolery, T. J. 1984. EQ6, A Computer Program for Reaction-Path Modeling of Aqueous Geochemical Systems: User's Guide and Documentation. UCRL-5l (Draft), Lawrence Livermore National Laboratory, University of California, Livermore, California.

Zhorov, V. A., A. A. Bezborodov, and N. I. Popov, 1976. "Predominance Area Diagrams and Equilibrium Ratios of Inorganic Forms of Cobalt and Nickel In Oceanic Water." Okeanol. 16:808-814. (Cited by Cosovic et a1. 1982.) 
APPENDIX A

REACTIONS INVOLVING AQUEOUS SPECIES AND

SOLID PHASES OF ANTIMONY, CERIUM, CESIUM, AND COBALT 
IABLE A.1. Reactions Involving Antimony Aqueous Species

ID Number

7403300

7403301

7402700

7402702

7407300

7407410
$\Delta H_{r, 298}^{0}$

$\mathrm{kJ} / \mathrm{mo}$

NA

NA

NA

NA

$-316.64$

NA $\underline{\log K} 298$

$+1.180$

$-11.803$

$+6.180$

$+6.182$

$+49.287$

$-29.37$

TABLE A.2. Reactions Involving Antimony Solid Phases

\begin{tabular}{|c|c|}
\hline In Number & Reaction \\
\hline 1074000 & $2 \mathrm{Sb}(\mathrm{OH})_{3}^{\mathrm{O}}+3 \mathrm{HS}^{-}+3 \mathrm{H}^{+} \rightleftarrows \mathrm{Sb}_{2} \mathrm{~S}_{3}+6 \mathrm{H}_{2} \mathrm{O}$ \\
\hline 2074000 & $2 \mathrm{Sb}(\mathrm{OH})_{3}^{\mathrm{O}}+\mathrm{Sb}_{2} \mathrm{O}_{3}+3 \mathrm{H}_{2} \mathrm{O}$ \\
\hline 2074001 & 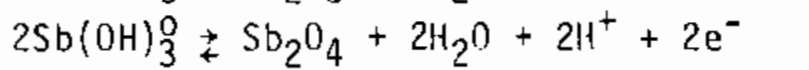 \\
\hline 2074100 & $2 \mathrm{Sb}(\mathrm{OH}) \frac{-}{6}+2 \mathrm{H}^{+} \underset{\mathrm{Sb}}{2} \mathrm{O}_{5}+7 \mathrm{H}_{2} \mathrm{O}$ \\
\hline 2074002 & $4 \mathrm{Sb}(\mathrm{OH})_{3}^{\mathrm{O}} \rightarrow \mathrm{Sb}_{4} \mathrm{O}_{6}+6 \mathrm{H}_{2} \mathrm{O}$ (cubic) \\
\hline 2074003 & $4 \mathrm{Sb}(\mathrm{OH})_{3}^{\mathrm{O}}+\mathrm{Sb}_{4} \mathrm{O}_{6}+6 \mathrm{H}_{2} \mathrm{O}$ (orthorhombic) \\
\hline 2074004 & $\mathrm{Sb}(\mathrm{OH})_{3}^{\mathrm{O}} \rightarrow \mathrm{Sb}(\mathrm{OH})_{3}(\mathrm{solid})$ \\
\hline 4174000 & $\mathrm{Sb}(\mathrm{OH})_{3}^{\mathrm{O}}+3 \mathrm{Cl}^{-}+3 \mathrm{H}^{+} \rightleftarrows \mathrm{SbCl}_{3}+3 \mathrm{H}_{2} \mathrm{O}$ \\
\hline 4074000 & $\mathrm{Sb}(\mathrm{OH})_{3}^{\mathrm{O}}+3 \mathrm{Br}^{-}+3 \mathrm{H}^{+} \rightarrow \mathrm{SbBr}_{3}+3 \mathrm{H}_{2} \mathrm{O}$ \\
\hline
\end{tabular}

\begin{tabular}{|c|c|}
\hline $\begin{array}{r}\Delta H_{r}^{0}, 298 \\
k J\} \text { mol } \\
\end{array}$ & $\underline{\log }_{298}$ \\
\hline-239.91 & +60.134 \\
\hline-18.79 & +8.481 \\
\hline+68.07 & -3.403 \\
\hline NA & +19.654 \\
\hline-61.09 & +19.661 \\
\hline-37.59 & +17.016 \\
\hline$N A$ & +2.355 \\
\hline+35.20 & -0.592 \\
\hline+21.23 & -1.053 \\
\hline
\end{tabular}

TABLE A.3. Reactions Involving Cerium Aqueous Species

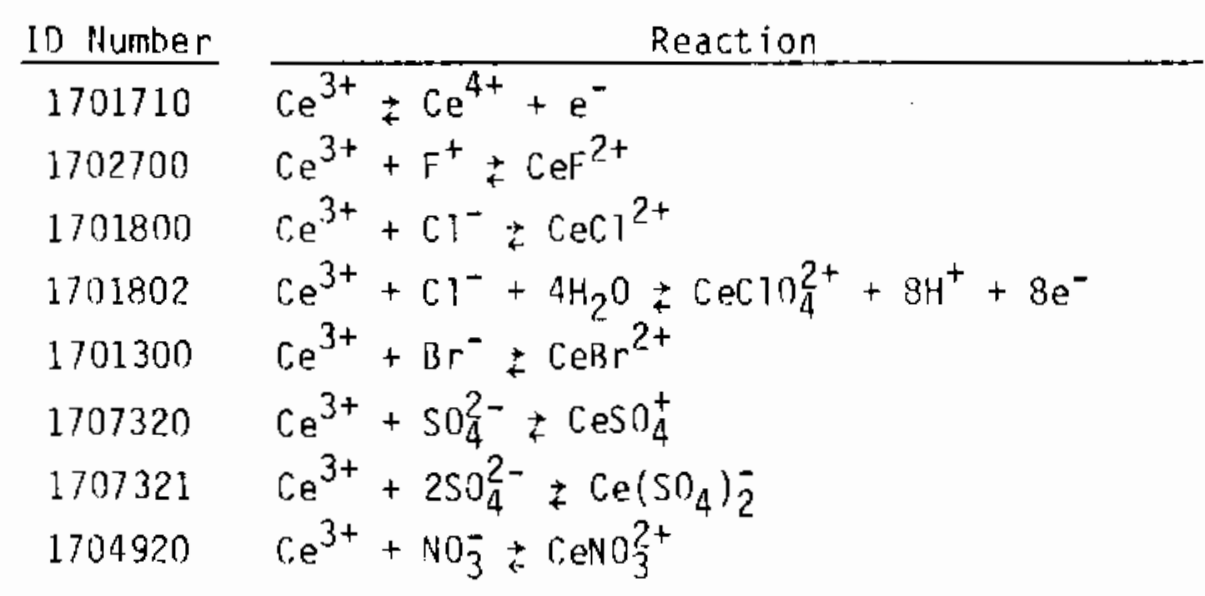

\begin{tabular}{crr}
$\begin{array}{rr}\Delta H_{r}^{0}, 298 \\
k J 01\end{array}$ & & Log $K_{298}$ \\
\hline+159.00 & & -29.467 \\
$N A$ & & +3.477 \\
+22.68 & & -0.804 \\
+1131.70 & & -185.866 \\
$N A$ & & +0.605 \\
+14.57 & & +3.446 \\
+21.53 & +5.071 \\
NA & +1.080
\end{tabular}


TABLE A.4. Reactions Involving Cerium Solid Phases

\begin{tabular}{|c|c|c|c|}
\hline In Number & Reaction & $\begin{array}{r}\Delta \mathrm{H}_{\mathrm{r}}^{0}, 298 \\
\mathrm{~kJ} / \mathrm{mo} \\
\end{array}$ & Log K $K_{298}$ \\
\hline 2017000 & $\mathrm{Ce}^{3+}+2 \mathrm{H}_{2} \mathrm{O}+\mathrm{CeO}_{2}$ (cerianite) $+4 \mathrm{H}^{+}+\mathrm{e}^{-}$ & +179.18 & -21.198 \\
\hline 2017001 & $2 \mathrm{Ce}^{3+}+3 \mathrm{H}_{2} \mathrm{O} \neq \mathrm{Ce}_{2} \mathrm{O}_{3}+6 \mathrm{H}^{+}$ & +453.69 & -60.901 \\
\hline 4117000 & $\mathrm{Ce}^{3+}+3 \mathrm{Cl}^{-} \neq \mathrm{CeCl}_{3}$ & +143.94 & -15.429 \\
\hline 4317002 & $\mathrm{Ce}^{3+}+31^{-}+11 \mathrm{H}_{2} \mathrm{O}+\mathrm{Ce}\left(\mathrm{IO}_{3}\right)_{3} \cdot 2 \mathrm{H}_{2} \mathrm{O}+18 \mathrm{H}^{+}+18 \mathrm{e}^{-}$ & NA & -324.850 \\
\hline 1017000 & $\mathrm{Ce}^{3+}+\mathrm{HS}^{-}+\mathrm{e}^{-} \ddagger \mathrm{CeS}+\mathrm{H}^{+}$ & +254.37 & -36.518 \\
\hline $54170 \cap 1$ & $\mathrm{Ce}^{3+}+\mathrm{Cr}(\mathrm{OH})_{2}^{+}+\mathrm{H}_{2} \mathrm{O} \rightarrow \mathrm{CeCrO}_{3}+4 \mathrm{H}^{+}$ & +183.81 & -17.310 \\
\hline 6017001 & $2 \mathrm{Ce}^{3+}+3 \mathrm{SO}_{4}^{2-}+8 \mathrm{H}_{2} \mathrm{O}+\mathrm{Ce}_{2}\left(\mathrm{SO}_{4}\right)_{3} \cdot 8 \mathrm{H}_{2} \mathrm{O}$ & $N A$ & +8.653 \\
\hline
\end{tabular}

TABLE A.5. Reactions Involving Cesium Aqueous Species

$\frac{\text { In Number }}{2201800} \frac{\text { Reaction }}{\mathrm{Cs}^{+}+\mathrm{Cl}^{-}+\mathrm{CsCl}^{\circ}}-\frac{\Delta \mathrm{KJ}_{\mathrm{r}}^{0} \text { mol }}{\mathrm{NA}} \frac{\log \mathrm{K}_{298}}{-0.34}$


IABLE A.6. Reactions Involving Cesium Solid Phases

\begin{tabular}{|c|c|c|c|}
\hline ID Number & Reaction & $\begin{array}{l}\Delta H_{r}^{0}, 298 \\
\mathrm{~kJ} / \mathrm{mol}]\end{array}$ & $\log _{293}$ \\
\hline 2022001 & $2 \mathrm{Cs}^{+}+\mathrm{H}_{2} \mathrm{O} \rightleftarrows \mathrm{Cs}_{2} \mathrm{O}+2 \mathrm{H}^{+}$ & +455.10 & -89.742 \\
\hline 2022002 & $\mathrm{Cs}^{+}+\mathrm{H}_{2} \mathrm{O} \rightleftarrows \mathrm{CSOH}+\mathrm{H}^{+}$ & +127.14 & -27.715 \\
\hline 4222000 & $\mathrm{CS}^{+}+\mathrm{F}^{-} \rightleftarrows \mathrm{CSF}$ & +39.85 & -8.401 \\
\hline 4222001 & $\mathrm{CS}^{+}+\mathrm{H}^{+}+2 \mathrm{~F}^{-} \nsucceq \mathrm{CSHF}_{2}$ & +4.916 & +0.658 \\
\hline 4122001 & $\mathrm{Cs}^{+}+\mathrm{Cl}^{-} \rightleftarrows \mathrm{CsCl}$ & -17.92 & -1.481 \\
\hline 4122003 & $\mathrm{Cs}^{+}+\mathrm{Cl}^{-}+3 \mathrm{H}_{2} \mathrm{O} \rightarrow \mathrm{CsClO}_{3}+6 \mathrm{H}^{+}+6 \mathrm{e}^{-}$ & +870.90 & -144.816 \\
\hline 4122004 & $\mathrm{Cs}^{+}+\mathrm{Cl}^{-}+4 \mathrm{H}_{2} \mathrm{O} \neq \mathrm{CsC} 10_{4}+8 \mathrm{H}^{+}+8 \mathrm{e}^{-}$ & +1125.35 & -185.248 \\
\hline 4022001 & $\mathrm{Cs}^{+}+\mathrm{Br}^{-} \rightleftarrows \mathrm{CsBr}$ & -26.27 & $-0.76 ?$ \\
\hline 4022003 & $\mathrm{Cs}^{+}+3 \mathrm{Br}^{-}+\mathrm{CsBr}_{3}+2 \mathrm{e}^{-}$ & +189.91 & -34.834 \\
\hline 4022004 & $\mathrm{Cs}^{+}+\mathrm{Br}^{-}+3 \mathrm{H}_{2} \mathrm{O}+\mathrm{CsBrO}_{3}+6 \mathrm{H}^{+}+6 \mathrm{e}^{-}$ & +861.22 & -144.067 \\
\hline 4322001 & $\mathrm{Cs}^{+}+\mathrm{I}^{-} \rightleftarrows \mathrm{CsI}$ & -31.66 & -0.538 \\
\hline 4322003 & $\mathrm{Cs}^{+}+3 \mathrm{I}^{-}+\mathrm{CsI}_{3}+2 \mathrm{e}^{-}$ & +66.82 & -16.241 \\
\hline 4322004 & $\mathrm{CS}^{+}+4 \mathrm{I}^{-} \neq \mathrm{CSI}_{4}+3 \mathrm{e}^{-}$ & +119.95 & -24.826 \\
\hline 4322005 & $\mathrm{Cs}^{+}+\mathrm{I}^{-}+3 \mathrm{H}_{2} \mathrm{O}_{+} \rightarrow \mathrm{CSIO}_{3}+6 \mathrm{H}^{+}+6 \mathrm{e}^{-}$ & NA & -108.851 \\
\hline 4322006 & $\mathrm{CS}^{+}+\mathrm{I}^{-}+4 \mathrm{H}_{2} \mathrm{O}^{0} \neq \mathrm{CSIO}_{4}+8 \mathrm{H}^{+}+8 \mathrm{e}^{-}$ & NA & -159.713 \\
\hline 4022005 & $\mathrm{Cs}^{+}+\mathrm{I}^{-}+2 \mathrm{Br}^{-} \rightarrow \mathrm{CSIBr}_{2}+2 \mathrm{e}^{-}$ & NA & -22.626 \\
\hline 4322007 & $\mathrm{Cs}^{+}+2 \mathrm{I}^{-}+\mathrm{Br}^{-} \nsucceq \mathrm{CSI} 2^{B r}+2 \mathrm{e}^{-}$ & +96.27 & -20.020 \\
\hline 4322008 & $\mathrm{CS}^{+}+\mathrm{I}^{-}+\mathrm{Br}^{-}+\mathrm{F}^{-}+\mathrm{CSIBrF}+2 \mathrm{e}^{-}$ & NA & -32.135 \\
\hline 4322009 & $\mathrm{Cs}^{+}+\mathrm{I}^{-}+\mathrm{Br}^{-}+\mathrm{Cl}^{-}+\mathrm{CSIBrCl}+2 \mathrm{e}^{-}$ & NA & -23.734 \\
\hline 6022001 & $2 \mathrm{Cs}^{+}+\mathrm{SO}_{4}^{2-} \neq \mathrm{Cs}_{2} \mathrm{SO}_{4}$ & -17.67 & -0.743 \\
\hline 5122001 & $\mathrm{Cs}^{+}+\mathrm{NO}_{3}^{-} \rightleftarrows \mathrm{CsNO}_{3}^{+}$ & -40.57 & +0.622 \\
\hline 5022001 & $2 \mathrm{Cs}^{+}+\mathrm{CO}_{3}^{2-} \div \mathrm{Cs}_{2} \mathrm{CO}_{3}$ & +53.50 & -9.979 \\
\hline 6022002 & $\mathrm{CS}^{+}+\mathrm{Al}^{3+}+2 \mathrm{SO}_{4}^{2-}+12 \mathrm{H}_{2} \mathrm{O} \underset{\leftarrow}{ } \mathrm{CSA} 1\left(\mathrm{SO}_{4}\right)_{2} \cdot 12 \mathrm{H}_{2} \mathrm{O}$ & -57.31 & +9.058 \\
\hline 4122005 & $\mathrm{Cs}^{+}+\mathrm{Cu}^{2+}+3 \mathrm{Cl}^{-} \neq \mathrm{CsCuCl}_{3}$ & -5.14 & +1.291 \\
\hline 7522000 & $2 \mathrm{Cs}^{+}+\mathrm{UO}_{2}^{2+}+2 \mathrm{H}_{2} \mathrm{O} \neq \mathrm{Cs}_{2} \mathrm{HO}_{4}+4 \mathrm{H}^{+}$ & +186.55 & -37.356 \\
\hline 3022003 & $2 \mathrm{C}, 5^{+}+2 \mathrm{CrO}_{4}^{2-}+2 \mathrm{H}^{+} \rightleftarrows \mathrm{Cs}_{2} \mathrm{Cr}_{2} \mathrm{O}_{7}+\mathrm{H}_{2} \mathrm{O}$ & -93.51 & +17.383 \\
\hline 5222000 & $\mathrm{CS}^{+}+\mathrm{H}_{3} \mathrm{BO}_{3} \rightarrow \mathrm{CSBO}_{2}+\mathrm{H}_{2} \mathrm{O}+\mathrm{H}^{+}$ & +72.13 & -18.968 \\
\hline 5222001 & $\mathrm{Cs}^{+}+\mathrm{H}_{3} \mathrm{BO}_{3}+4 \mathrm{Cl}^{-}+3 \mathrm{H}^{+} \rightleftarrows \mathrm{CsBCl}_{4}+3 \mathrm{H}_{2} \mathrm{O}$ & +199.39 & -43.350 \\
\hline
\end{tabular}


TABLE A.7. Reactions Involving Cobalt Aqueous Species

\begin{tabular}{|c|c|c|c|}
\hline In Number & Reaction & $\begin{array}{r}\Delta H_{\mathrm{r}}^{0} \\
\mathrm{~kJ} / \mathrm{mol} \\
\end{array}$ & $\underline{\log K_{298}}$ \\
\hline 2012000 & $\mathrm{CO}^{3+} \mathrm{e}^{-} \rightleftarrows \mathrm{Co}^{2+}$ & NA & +33.01 \\
\hline 2003300 & $\mathrm{Co}^{2}+\mathrm{H}_{2} \mathrm{O} \neq \mathrm{COOH}^{+}+\mathrm{H}^{+}$ & NA & -9.67 \\
\hline 2003301 & $\mathrm{CO}^{2+}+2 \mathrm{H}_{2} \mathrm{O} Z \mathrm{CO}(\mathrm{OH})_{2}^{0}+2 \mathrm{H}^{+}$ & NA & -18.76 \\
\hline $200330 ?$ & $\mathrm{Co}^{2+}+3 \mathrm{H}_{2} \mathrm{O} \rightarrow \mathrm{CO}(\mathrm{OH})_{3}^{-}+3 \mathrm{H}^{+}$ & NA & -32.23 \\
\hline 2003303 & $\mathrm{CO}^{2+}+4 \mathrm{H}_{2} \mathrm{O} \rightarrow \mathrm{Co}(\mathrm{OH})_{4}^{2-}+4 \mathrm{H}^{+}$ & NA & -45.78 \\
\hline 2003304 & $2 \mathrm{Co}^{2+}+\mathrm{H}_{2} \mathrm{O} \rightleftarrows \mathrm{CO}_{2} \mathrm{OH}^{3+}+\mathrm{H}^{+}$ & NA & -11.28 \\
\hline 2003305 & $\mathrm{CO}^{2+}+2 \mathrm{H}_{2} \mathrm{O}+\mathrm{HCOO}_{2}^{-}+3 \mathrm{H}^{+}$ & NA & -21.24 \\
\hline 2003306 & $4 \mathrm{Co}^{2+}+4 \mathrm{H}_{2} \mathrm{O} \rightleftarrows \mathrm{CO}_{4}(\mathrm{OH})_{4}^{4+}+4 \mathrm{H}^{+}$ & $\mathrm{NA}$ & -30.32 \\
\hline 2002700 & $\mathrm{Co}^{2+}+\mathrm{F}^{-} \rightleftarrows \mathrm{CoF}^{+}$ & NA & +0.41 \\
\hline 2001400 & $\mathrm{Co}^{2+}+\mathrm{CO}_{3}^{2-} \rightleftarrows \mathrm{CoCO}_{3}^{0}$ & NA & +3.17 \\
\hline 2001401 & $\mathrm{CO}^{2+}+\mathrm{CO}_{3}^{2-}+\mathrm{H}^{+} \rightleftarrows \mathrm{CoHCO}_{3}^{+}$ & NA & +11.72 \\
\hline 2007300 & $\mathrm{Co}^{2+}+\mathrm{HS}^{-} z \mathrm{COHS}^{+}$ & NA & +6.51 \\
\hline 2007301 & $\mathrm{CO}^{2+}+2 \mathrm{HS}^{-} \rightleftarrows \mathrm{Co}(\mathrm{HS})_{2}^{0}$ & NA & +8.77 \\
\hline 2007350 & $\mathrm{Co}^{2+}+\mathrm{S}_{2} \mathrm{O}_{3}^{2-}+\mathrm{CoS}_{2} \mathrm{O}_{3}^{0}$ & $N A$ & -16.86 \\
\hline 2005800 & $\mathrm{CO}^{2+}+\mathrm{PO}_{4}^{3-}+\mathrm{H}^{+}+\mathrm{COHPO}_{4}^{\circ}$ & $N A$ & +14.53 \\
\hline 2005801 & $\mathrm{Co}^{2+}+2 \mathrm{PO}_{4}^{3-}+2 \mathrm{H}^{+} \rightleftarrows \mathrm{COP}_{2} \mathrm{O}_{7}^{2-}+\mathrm{H}_{2} \mathrm{O}$ & NA & +26.89 \\
\hline 2004900 & $\mathrm{Co}^{2+}+\mathrm{NH}_{4}^{+} \rightleftarrows \mathrm{CoNH}_{3}^{2+}+\mathrm{H}^{+}$ & +46.27 & -7.25 \\
\hline 2004901 & $\mathrm{CO}^{2+}+2 \mathrm{NH}_{4}^{+} \neq \mathrm{Co}\left(\mathrm{NH}_{3}\right)_{2}^{2+}+2 \mathrm{H}^{+}$ & N.A & -15.01 \\
\hline 2004902 & $\mathrm{CO}^{2+}+3 \mathrm{NH}_{4}^{+} \neq \mathrm{CO}\left(\mathrm{NH}_{3}\right)_{3}^{2+}+3 \mathrm{H}^{+}$ & NA & -23.44 \\
\hline 2004903 & $\mathrm{CO}^{2+}+4 \mathrm{NH}_{4}^{+}+\mathrm{Co}\left(\mathrm{NH}_{3}\right)_{4}^{2+}+4 \mathrm{H}^{+}$ & NA & -32.08 \\
\hline 2004904 & $\mathrm{Co}^{2+}+5 \mathrm{NH}_{4}^{+} \rightleftarrows \mathrm{Co}\left(\mathrm{NH}_{3}\right)_{5}^{2+}+5 \mathrm{H}^{+}$ & $N A$ & -41.23 \\
\hline 2014907 & $\mathrm{Co}^{3+}+6 \mathrm{NH}_{4}^{+} \rightleftarrows \mathrm{Co}\left(\mathrm{NH}_{3}\right)_{6}^{3+}+6 \mathrm{H}^{+}$ & +122.63 & -32.56 \\
\hline 2014900 & $\mathrm{CO}^{3+}+5 \mathrm{NH}_{4}^{+}+\mathrm{NO}_{2}^{-} \pm\left[\mathrm{Co}\left(\mathrm{NH}_{3}\right)_{5} \mathrm{NO}_{2}\right]^{2+}+5 \mathrm{H}^{+}$ & +65.52 & -23.33 \\
\hline 2014901 & $\mathrm{Co}^{3+}+6 \mathrm{NH}_{4}^{+}+\mathrm{H}_{2} \mathrm{O} \Rightarrow\left[\mathrm{Co}\left(\mathrm{NH}_{3}\right)_{5}\right] \mathrm{OH}^{2+}+7 \mathrm{H}^{+}$ & NA & -43.69 \\
\hline 2014902 & $\mathrm{CO}^{3+}+5 \mathrm{NH}_{4}^{+}+\mathrm{Cl}^{-} \rightleftarrows\left[\mathrm{Co}\left(\mathrm{NH}_{3}\right)_{5}\right] \mathrm{Cl}^{2+}+5 \mathrm{H}^{+}$ & +113.36 & -18.03 \\
\hline 2014903 & $\mathrm{CO}^{3+}+6 \mathrm{NH}_{4}^{+}+\mathrm{Cl}^{-} \neq\left[\mathrm{Co}\left(\mathrm{NH}_{3}\right)_{6}\right] \mathrm{Cl}^{2+}+6 \mathrm{H}^{+}$ & +104.36 & -34.01 \\
\hline 2014904 & $\mathrm{Co}^{3+}+6 \mathrm{NH}_{4}^{+}+\mathrm{Br}^{-} \div\left[\mathrm{Co}\left(\mathrm{NH}_{3}\right)_{6}\right] \mathrm{Br}^{2+}+6 \mathrm{H}^{+}$ & +110.66 & -39.99 \\
\hline 2014905 & $\mathrm{Co}^{3+}+6 \mathrm{NH}_{4}^{+}+\mathrm{I}^{-} \rightleftarrows\left[\mathrm{Co}\left(\mathrm{NH}_{3}\right)_{6}\right] \mathrm{I}^{2+}+6 \mathrm{H}^{+}$ & +115.52 & -33.59 \\
\hline 2014906 & $\mathrm{CO}^{3+}+6 \mathrm{NH}_{4}^{+}+\mathrm{SO}_{4}^{2-} \rightleftarrows\left[\mathrm{CO}\left(\mathrm{NH}_{3}\right)_{6}\right] \mathrm{SO}_{4}^{+}+6 \mathrm{H}^{+}$ & +124.39 & -29.16 \\
\hline
\end{tabular}


TABLF A.8. Reactions Involving Cobalt Solid Phases

\begin{tabular}{|c|c|c|c|}
\hline ID Number & Reaction & $\begin{array}{r}\Delta H_{\mathrm{r}}^{0}, 298 \\
\mathrm{~kJ} / \mathrm{mol} \\
\end{array}$ & $\underline{\log } K_{29}$ \\
\hline 0020000 & $\mathrm{Co}^{2+}+2 \mathrm{e}^{-} \neq \mathrm{Co}(\mathrm{al}$ pha, hexàgonal $)$ & +58.20 & -9.53 \\
\hline 2020001 & $\mathrm{Co}^{2+}+\mathrm{H}_{2} \mathrm{O} \neq \mathrm{COO}+2 \mathrm{H}^{+}$ & +106.08 & -13.56 \\
\hline 2020002 & $\mathrm{Co}^{2+}+2 \mathrm{H}_{2} \mathrm{O} \pm \mathrm{Co}(\mathrm{OH})_{2}(\mathrm{blue} \mathrm{ppt.})+2 \mathrm{H}^{+}$ & NA & -13.77 \\
\hline 2020003 & $\mathrm{CO}^{2+}+2 \mathrm{H}_{2} \mathrm{O} \neq \mathrm{Co}(\mathrm{OH})_{2}$ (pink ppt. $)+2 \mathrm{H}^{+}$ & +90.12 & -13.03 \\
\hline 2020004 & $\mathrm{Co}^{2+}+2 \mathrm{H}_{2} \mathrm{O} \neq \mathrm{Co}(\mathrm{OH})_{2}$ (pink ppt., aged) $+2 \mathrm{H}^{+}$ & NA & -12.37 \\
\hline 2020005 & $\mathrm{CO}^{2+}+2 \mathrm{H}_{2} \mathrm{O} \neq \mathrm{Co}(\mathrm{OH})_{2}($ transvaalite $)+2 \mathrm{H}^{+}$ & NA & -12.08 \\
\hline 2020100 & $\mathrm{Co}^{3+}+3 \mathrm{H}_{2} \mathrm{O} \neq \mathrm{CO}(\mathrm{OH})_{3}+3 \mathrm{H}^{+}$ & +34.96 & +3.34 \\
\hline 3020100 & $3 \mathrm{Co}^{2+}+4 \mathrm{H}_{2} \mathrm{O}+\mathrm{Co}_{3} \mathrm{O}_{4}$ (cobalt spinel) $+8 \mathrm{H}^{+}+2 \mathrm{e}^{-}$ & +426.73 & -59.40 \\
\hline 2020101 & $\mathrm{CO}^{3+}+2 \mathrm{H}_{2} \mathrm{O} \neq \mathrm{COOOH}$ (heterogenite) $+3 \mathrm{H}^{+}$ & NA & +10.23 \\
\hline 4220000 & $\mathrm{Co}^{2+}+2 \mathrm{~F}^{-} \neq \mathrm{CoF}_{2}$ & +36.86 & +5.15 \\
\hline 4120000 & $\mathrm{Co}^{2+}+2 \mathrm{Cl}^{-}+\mathrm{CoCl}_{2}$ & +79.81 & -8.25 \\
\hline 4120001 & $\mathrm{Co}^{2+}+2 \mathrm{Cl}^{-}+\mathrm{H}_{2} \mathrm{O} \neq \mathrm{CoCl}_{2} \cdot \mathrm{H}_{2} \mathrm{O}$ & +63.14 & -4.73 \\
\hline 4120002 & $\mathrm{CO}^{2+}+2 \mathrm{Cl}^{-}+2 \mathrm{H}_{2} \mathrm{O} \neq \mathrm{CoCl}_{2} \cdot 2 \mathrm{H}_{2} \mathrm{O}$ & +41.03 & -4.65 \\
\hline 4120003 & $\mathrm{Co}^{2+}+2 \mathrm{Cl}^{-}+6 \mathrm{H}_{2} \mathrm{O} \neq \mathrm{CoCl}_{2} \cdot 6 \mathrm{H}_{2} \mathrm{O}$ & -8.09 & -2.56 \\
\hline 7020000 & $\mathrm{CO}^{2+}+\mathrm{PO}_{4}^{3-}+\mathrm{H}^{+} \neq \mathrm{CoHPO}_{4}$ & NA & +19.06 \\
\hline 7020001 & $3 \mathrm{CO}^{2+}+2 \mathrm{PO}_{4}^{3-} \neq \mathrm{CO}_{3}\left(\mathrm{PO}_{4}\right)_{2}$ & NA & +34.67 \\
\hline 5020000 & $\mathrm{Co}^{2+}+\mathrm{CO}_{3}^{2-} \neq \mathrm{CoCO}_{3}$ (spherocobaltite) & +19.25 & +10.14 \\
\hline 1020000 & $\mathrm{Co}^{2+}+\mathrm{HS}^{-} \neq \operatorname{CoS}($ jaipurite $)+\mathrm{H}^{+}$ & -20.46 & +9.29 \\
\hline 1020001 & $\mathrm{CO}^{2+}+2 \mathrm{HS}^{-} \neq \operatorname{CoS}_{2}$ (cattierite) $+2 \mathrm{H}^{+}+2 \mathrm{e}^{-}$ & -59.79 & +20.20 \\
\hline 1020002 & $3 \mathrm{Co}^{2+}+4 \mathrm{HS}^{-} \neq \mathrm{CO}_{3} \mathrm{~S}_{4}(1$ innaeite $)+4 \mathrm{H}^{+}+2 \mathrm{e}^{-}$ & -114.10 & +40.99 \\
\hline 1020003 & $9 \mathrm{CO}^{2+}+8 \mathrm{HS}^{-}+2 \mathrm{e}^{-} \neq \mathrm{Cog}_{8}$ (cobalt pentlandite) $+8 \mathrm{H}^{+}$ & -186.65 & +77.36 \\
\hline 6020000 & $\mathrm{Co}^{2+}+\mathrm{SO}_{4}^{2-}+\mathrm{CoSO}_{4}$ & +79.20 & -2.91 \\
\hline 6020001 & $\mathrm{Co}^{2+}+\mathrm{SO}_{4}^{2-}+7 \mathrm{H}_{2} \mathrm{O} \neq \mathrm{CoSO}_{4} \cdot 7 \mathrm{H}_{2} \mathrm{O}$ (bieberite) & -11.65 & +2.46 \\
\hline 6020002 & $\mathrm{CO}^{2+}+\mathrm{SO}_{4}^{2-}+6 \mathrm{H}_{2} \mathrm{O} \neq \mathrm{CoSO}_{4} \cdot 6 \mathrm{H}_{2} \mathrm{O}$ & -1.17 & +2.31 \\
\hline 6020003 & $\mathrm{CO}^{2+}+\mathrm{SO}_{4}^{2-}+\mathrm{H}_{2} \mathrm{O}+\mathrm{CoSO}_{4} \cdot \mathrm{H}_{2} \mathrm{O}$ & +52.57 & +1.18 \\
\hline 7220002 & $\mathrm{Co}^{2+}+\mathrm{H}_{3} \mathrm{ASO}_{4}^{\circ}+5 \mathrm{H}^{+}+7 \mathrm{e}^{-} \neq \mathrm{COAs}$ (modderite) $+4 \mathrm{H}_{2} \mathrm{O}$ & -233.68 & +31.12 \\
\hline 7220003 & $\mathrm{CO}^{2+}+2 \mathrm{H}_{3} \mathrm{AsO}_{4}^{\circ}+10 \mathrm{H}^{+}+12 \mathrm{e}^{-} \neq \mathrm{COAs}_{2}$ (safflorite) $+8 \mathrm{H}_{2} \mathrm{O}$ & -506.72 & +71.41 \\
\hline 7220000 & $3 \mathrm{Co}^{2+}+2 \mathrm{H}_{3} \mathrm{AsO}_{4}^{O} \neq \mathrm{CO}_{3}\left(\mathrm{AsO}_{4}\right)_{2}+6 \mathrm{H}^{+}$ & NA & -13.05 \\
\hline 7220001 & $3 \mathrm{CO}^{2+}+2 \mathrm{H}_{3} \mathrm{AsO}_{4}^{\mathrm{O}}+8 \mathrm{H}_{2} \mathrm{O} \neq \mathrm{CO}_{3}\left(\mathrm{AsO}_{4}\right)_{2} * 8 \mathrm{H}_{2} \mathrm{O}$ (erythrite) $+6 \mathrm{H}^{+}$ & NA & -12.99 \\
\hline 5120100 & $\mathrm{CO}^{3+}+5 \mathrm{NH}_{4}^{+}+\mathrm{NO}_{2}^{-}+2 \mathrm{NO}_{3}^{-} \neq\left[\mathrm{Co}\left(\mathrm{NH}_{3}\right)_{5} \mathrm{NO}_{2}\right]\left(\mathrm{NO}_{3}\right)_{2}+5 \mathrm{H}^{+}$ & +4.94 & -19.31 \\
\hline 4120100 & $\mathrm{CO}^{3+}+5 \mathrm{NH}_{4}^{+}+3 \mathrm{Cl}-\left[\mathrm{Co}\left(\mathrm{NH}_{3}\right)_{5} \mathrm{Cl}^{-}\right] \mathrm{Cl}_{2}+5 \mathrm{H}^{+}$ & +58.40 & -13.09 \\
\hline 4120101 & $\mathrm{Co}^{3+}+5 \mathrm{NH}_{4}^{+}+\mathrm{H}_{2} \mathrm{O}+3 \mathrm{Cl}^{-} \neq\left[\mathrm{Co}\left(\mathrm{NH}_{3}\right)_{5} \mathrm{H}_{2} \mathrm{O}\right] \mathrm{Cl}_{3}+5 \mathrm{H}^{+}$ & +73.53 & -20.42 \\
\hline 4120102 & $\mathrm{CO}^{3+}+6 \mathrm{NH}_{4}^{+}+3 \mathrm{ClO} 0_{4}^{-} \neq\left[\mathrm{Co}\left(\mathrm{NH}_{3}\right)_{6}\right]\left(\mathrm{ClO}_{4}\right)_{3}+6 \mathrm{H}^{+}$ & +60.83 & -25.87 \\
\hline 4020100 & $\mathrm{CO}^{3+}+6 \mathrm{NH}_{4}^{+}+3 \mathrm{Br} \mathrm{r}^{-} \neq\left[\mathrm{Co}\left(\mathrm{NH}_{3}\right)_{6}\right] \mathrm{Br}_{3}+6 \mathrm{H}^{+}$ & +69.15 & -26.99 \\
\hline 5120101 & $\mathrm{CO}^{3+}+6 \mathrm{NH}_{4}^{+}+3 \mathrm{NO}_{3}^{-} \neq\left[\mathrm{CO}\left(\mathrm{NH}_{3}\right)_{6}\right]\left(\mathrm{NO}_{3}\right)_{3}+6 \mathrm{H}^{+}$ & +47.66 & -26.64 \\
\hline
\end{tabular}


. 


\section{OISTRIBUTION}

No. of

Copies

OFFSITE

6 Geologic Repository Division DOE Office of Civilian

Radioactive Waste Management Forrestal Building

Washington, D.C. 20585

ATTN: C. R. Cooley, RW-4

J. R. Hilley

W. J. Purcell, RW-20

B. C. Rusche, RW-1

D. E. Shel or

R. Stein, RW-23

3 DOE Office of Defense Waste \&

Byproducts Management GTN

Washington, D.C. 20545

ATTN: R. K. Heusser

J. E. Lyt7e, DP-12

R. D. Walton, Jr., DP-123

6 DOE Office of Terminal Waste Disposal \& Remedial Action GTN

Washington, D.C. 20545

ATTN: J. A. Coleman, NE-25

T. W. McIntosh, NE-25

H. Stelling, NE-25

W. R. Voigt, NE-20

H. F. Walter, NE-25

J. B. Zorn, NE-25

3 DOE Idaho Operations office

550 second Street

Idaho Falls, ID 83401

ATTN: J. P. Hamric

S. Henchberger

J. E. Sol ecki

W. J. Brumley

DOE Savannah River Operations Office

P.0. Box A

Aiken, SC 29801
No. of

Copies

F. T. Fong

DOE San Francisco Operations

1333 Broadway

0akl and, CA 94612

M. R. Jugan

DOE Oak Ridge Operations office

P.0. Box E

Oak Ridge, TN 37830

E. Maestas

DOE West Valley Operations office

P.0. Box 191

West Valley, NY 14171

J. M. McGough

DOE Albuquerque Operations Office

P.0. Box 5400

Albuquerque, NM 87185

30 OOE Technical Information Center

C. S. Abrams

Argonne National Laboratory

P.0. Box 2528

Idaho Falls, ID 83401

M. J. Steindler

Argonne National Laboratory

9700 South Cass Avenue

Argonne, IL 60439

D. B. Shipp

Battelle Memorial Institute

office of Crystalline Repository Devel opment

9800 South Cass Avenue

Argonne, IL 60439 
No. of

Copies

3 Battelle Memorial Institute Project Management Division 505 King Avenue

Col umbus, $\mathrm{OH} 43201$

ATTN: W. A. Carbeiner

W. S. Madia

Technical Library

E. I. du Pont de Nemours Company

Savannah River Laboratory

Aiken, SC 29801

ATTN: M. D. Boersma

6 EG\&G Idaho

P.0. Box 1625

Idaho Fa11s, ID 83415

ATTN: E. A. Jennrich

D. Hal ford

J. Bradford

J. Logan

S. Minkin

T. Tates

R. Williams

Electric Power Research Institute

3412 Hillview Avenue

P.0. Box 10412

Palo Alto, CA 94304

J. L. Larocca, Chairman

Energy Research \& Development

Authority

Empire State Plaza

Albany, NY 12223

G. L. Sjoblom

Environmental Protection Agency

Office of Radiation Programs

401 M Street, S.W.

Washington, D.C. 20460

P. G. Hagen

Joint Integration office

Bldg. 3, 2nd Floor

2201 San Pedro N.E.

Albuquerque, NM 87110
No. of

Copies

D. T. Oakley, MS 671

Los Almos Scientific Laboratory P.0. Box 1663

Los Alamos, NM 87544

A. T. Clark

Division of Fuel Material Safety Nuclear Regulatory Commission

Washington, D.C. 20555

V. Stello

Office of the Executive Director for Operations

Mail Station 6209

Nuclear Regulatory Commission

Washington, 0.C. 20555

4 Oak Ridge National Laboratory

P.0. Box Y

Oak Ridge, TN 37830

ATTN: J. 0. Bl omeke

W. D. Burch

R. T. Jubin

L. J. Mezga

4 Sandia Laboratories

P.O. Box 5800

Albuquerque, NM 87185

ArTN: D. R. Anderson

R. W. Lynch

W. Weart

Technical Library

3 Technical Library

Los Al amos National Laboratory

Los Al amos, New Mexico 87545

3 Environmental Protection Agency

Technology Assessment Division

office of Radiation Programs

Washington, D.C. 20460

3 Technical Library

Argonne National Laboratory

Argonne, IL 60439 
No. of

Copies

3 Technical Library

Idaho National Engineering

Laboratory

Idaho Falls, 1083401

3 Technical Library

Savannah River National

Laboratory

Aiken, SC 29081

3 Technial Library

Oak Ridge National Laboratory

Oak Ridge, Tennessee 37830

2 National Academy of Sciences

National Research Council

2101 Constitution Avenue

Washington, D.C. 20418

J. R. Ber reth

Westinghouse Idaho Nuclear

Co., Inc.

P.0. Box 4000

Idaho Falls, ID 83401

West Valley Nuclear Services

Company

P.0. Box 191

West Valley, NY 14171

ATTN: J. M. Pope

3 S. R. Peterson

ICF Northwest

HAPO Building, 4th Floor

601 Williams Blvd.

Richl and, WA 99352

\section{ONSITE}

3 DOE Richl and Operations office

E. A. Bracken

N. T. Karagianes

J. D. White
No, of

Copies

12 Rockwell Hanford Operations

N. W. Kirch

R. N. Gurley

J. M. Henderson

H. E. McGuire

K. Owen

R. D. Prosser

I. E. Reep

R. J. Thompson

T. B. Venziano

D. D. Wodrich

R. D. Wojtasak

File Copy

UNC United Nuclear Industries

T. E, Dabrowski/W. J. Kyriazis

2 Westinghouse Hanford Company

R. E. Lerch

J. D. Watrous

50 Pacific Northwest Laboratory

C. R. Alien

W. W. Ballard, Jr.

M. P. Bergeron

W. $F$. Bonner

J. L. Buelt

H. C. Burkhol der

L. J. Criscenti

D. W. Dragnich

L. E. Eary

C. E. Elderkin

R. L. Erikson

J. S. Fruchter

M. J. Graham (2)

L. K. Grove

H. A. Haerer

J. M. Hales

M. S. Hanson

P. C. Hays

C. J. Hostet?er

J. H. Jarrett

E. A. Jenne

K. M. Krupka

Distr -3 
No. of

Copies

W. W. Laity

L. T. Lakey

J. M. Latkovich

W. J. Martin

E. D. MCCl anham,

J. L. McEl roy

J. E. Minor

W. J. Mitchell

B. E. Opitz (3)
No. of

Copies

J. A. Powe11

W. A. Ross

R. J. Serne

R. L. Skaggs (3)

J. A. Stottlemyre

J. L. Straal sund

R. E. Wi1dung

Publishing Coordination (2)

Technical Report Files (5) 